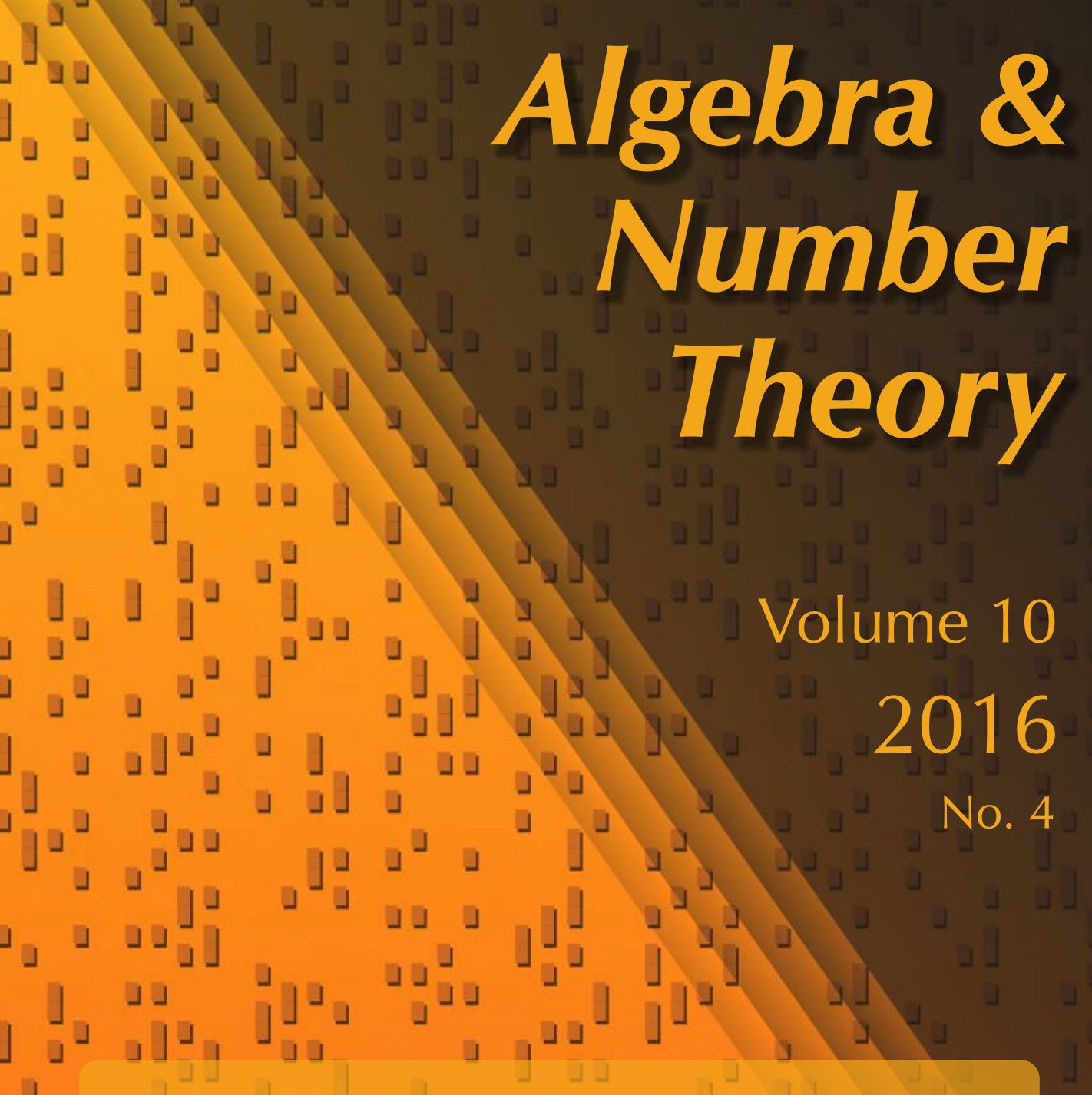

Analytic continuation on Shimura varieties with $\mu$-ordinary locus

\lrcorner

\footnotetext{
\lrcorner Stéphane Bijakowski
}

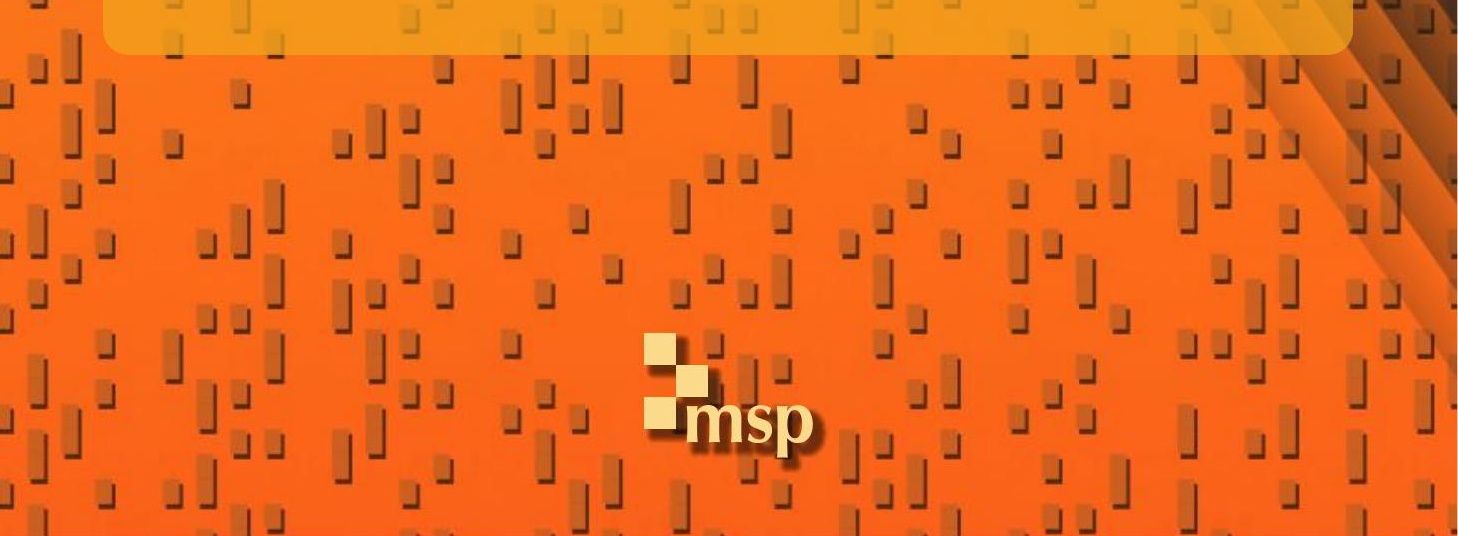




\title{
Analytic continuation on Shimura varieties with $\mu$-ordinary locus
}

\author{
Stéphane Bijakowski
}

\begin{abstract}
We study the geometry of unitary Shimura varieties without assuming the existence of an ordinary locus. We prove, by a simple argument, the existence of canonical subgroups on a strict neighborhood of the $\mu$-ordinary locus (with an explicit bound). We then define the overconvergent modular forms (of classical weight) as well as the relevant Hecke operators. Finally, we show how an analytic continuation argument can be adapted to this case to prove a classicality theorem, namely that an overconvergent modular form which is an eigenform for the Hecke operators is classical under certain assumptions.
\end{abstract}

Introduction

1. Shimura varieties of type (A)

846

2. Modular forms and Hecke operators

862

3. A classicality result

869

4. The case with several primes above $p$

Acknowledgement

884

References

885

\section{Introduction}

A modular form is defined as a global section of a certain sheaf on the modular curve. To study congruences between modular forms, one is led to introduce new objects, namely $p$-adic and overconvergent modular forms. These are sections of a sheaf on the ordinary locus of the modular curve and on a strict neighborhood of the ordinary locus, respectively. A lot of work has been done using these objects; one can for example construct families of overconvergent modular forms.

It is possible to generalize the definition of $p$-adic and overconvergent modular forms to other varieties, for example the Hilbert modular variety or the Siegel variety. The natural definition of an overconvergent modular form is a section of a certain sheaf on a strict neighborhood of the ordinary locus. In greater generality, one can consider Shimura varieties with a nonempty ordinary locus.

MSC2010: primary 11G18; secondary 11F55, $14 \mathrm{G} 35$.

Keywords: Shimura variety, overconvergent modular forms, $\mu$-ordinary locus, canonical subgroups. 
But for Shimura varieties without ordinary locus, this definition fails. Recall that, for Shimura varieties of PEL type, the criterion for the existence of an ordinary locus at $p$ is that $p$ splits completely in its reflex field. For Shimura varieties of PEL type (C) (associated to symplectic groups), the reflex field is $\mathbb{Q}$, so there is always an ordinary locus. But, if one considers Shimura varieties of type (A) (associated to unitary groups), then the reflex field may not be $\mathbb{Q}$ and the ordinary locus may be empty.

Let us look at an example. Let $F$ be a CM-field with totally real subfield $F_{0}$, and consider the special fiber of the Shimura variety associated to a unitary group with signature $\left(a_{\sigma}, b_{\sigma}\right)$ at each real place $\sigma$ of $F_{0}$. Suppose for simplicity that $p$ is a prime number inert in $F_{0}$ that splits as $\pi^{+} \pi^{-}$in $F$. The choice of $\pi^{+}$gives an order for the elements of the pair $\left(a_{\sigma}, b_{\sigma}\right)$ for each real place $\sigma$ of $F_{0}$. The existence of the ordinary locus at $p$ is then equivalent to the fact that there exist integers $a$ and $b$ such that $a_{\sigma}=a$ and $b_{\sigma}=b$ for each real place $\sigma$. The structure of the $p$-torsion of the abelian variety is then well known on the ordinary locus. In our setting, the $p$-divisible group $A\left[p^{\infty}\right]$ splits as $A\left[\left(\pi^{+}\right)^{\infty}\right] \oplus A\left[\left(\pi^{-}\right)^{\infty}\right]$, and $A\left[\left(\pi^{-}\right)^{\infty}\right]$ is the dual of $A\left[\left(\pi^{+}\right)^{\infty}\right]$. On the ordinary locus, the $p$-divisible group $A\left[\left(\pi^{+}\right)^{\infty}\right]$ is an extension of a multiplicative part of height $d a$ and an étale part of height $d b$ ( $d$ is the degree of $F_{0}$ over $\mathbb{Q}$ ). In particular, there exists a multiplicative subgroup $H_{a} \subset A\left[\pi^{+}\right]$of height $d a$. Actually, this property characterizes the ordinary locus: if there exists a multiplicative subgroup $H_{a}$ of height $h a$, then the abelian variety is ordinary at $p$. On the rigid space associated to the Shimura variety (i.e., the generic fiber of its formal completion along its special fiber), one can define the ordinary locus. There is still a multiplicative subgroup of $A\left[\pi^{+}\right]$of height $d a$ on this locus, and work of Fargues [2011] shows that this subgroup extends to a canonical subgroup on a strict neighborhood of the ordinary locus.

If the $\left(a_{\sigma}\right)$ are not equal to a certain integer $a$, then the ordinary locus is empty. There is always a special locus in the special fiber of the Shimura variety, called the $\mu$-ordinary locus, but the situation is more involved. Suppose we are in the same setting as before, and let us order the elements $a_{1} \leq a_{2} \leq \cdots \leq a_{d}$. Then the $\mu$-ordinary locus is characterized by the fact that the $p$-divisible group $A\left[\left(\pi^{+}\right)^{\infty}\right]$ has a filtration $0 \subset X_{1} \subset X_{2} \subset \cdots \subset X_{d+1}=A\left[\left(\pi^{+}\right)^{\infty}\right]$ such that $X_{i+1} / X_{i}$ is a $p$-divisible group of height $d\left(a_{i+1}-a_{i}\right)$ with its structure explicitly described (by convention we set $a_{0}=0$ and $\left.a_{d+1}=a_{d}+b_{d}\right)$. The fact that $A$ is $\mu$-ordinary is then also equivalent to the existence of subgroups $0 \subset H_{a_{1}} \subset \cdots \subset H_{a_{d}} \subset A\left[\pi^{+}\right]$, with $H_{a_{i}}$ of height $d a_{i}$ and the structure of $H_{a_{i+1}} / H_{a_{i}}$ explicitly described.

If one looks at the rigid space of the Shimura variety, then one can define the $\mu$-ordinary locus. There are several canonical subgroups in $A\left[\pi^{+}\right]$on this locus. We lack a good theory for these canonical subgroups, which should be analogous to the one given by Fargues [2011]. However, by simple arguments, one can prove the following fact: 
Theorem. On a strict neighborhood of the $\mu$-ordinary locus, there exist canonical subgroups $H_{a_{1}} \subset \cdots \subset H_{a_{d}}$ in $A\left[\pi^{+}\right]$. These subgroups are characterized by the fact that their degree (in the sense of [Fargues 2010]) is maximal among the subgroups of the same height of $A\left[\pi^{+}\right]$.

The proof is actually very simple: Let us consider $X_{\mathrm{Iw}}$, the variety with Iwahori level at $p$, and let $f: X_{\mathrm{Iw}} \rightarrow X$ be the projection. The $\mu$-ordinary locus is the image under $f$ of the locus where the subgroups $H_{a_{i}}$ are of maximal degree. Let $0<\varepsilon<\frac{1}{2}$, and consider the locus in $X_{\text {Iw }}$ where the degree of $H_{a_{i}}$ is bigger than the maximal degree minus $\varepsilon$. The image under $f$ of this locus is a strict neighborhood of the $\mu$-ordinary locus. The existence of canonical subgroups follows from the definition. Their uniqueness is a simple computation using the properties of the degree function (see Proposition 1.24).

If we consider the space $X_{\mathrm{Iw}}$, then one can call the locus where the degree of each $H_{a_{i}}$ is maximal the $\mu$-ordinary-multiplicative locus. It then makes sense to define an overconvergent modular form as the section of a certain sheaf on a strict neighborhood of the $\mu$-ordinary-multiplicative locus.

The Hecke algebra at $p$ acts both on the rigid space and on the space of modular forms. In the case of the existence of the ordinary locus, there is one relevant Hecke operator, parametrizing complements of the canonical subgroup. In the general case, there will be as many relevant Hecke operators as the number of canonical subgroups. We will denote by $U_{p, a_{i}}$ these Hecke operators. One can show that these operators increase the degrees of all the subgroups of $A\left[\pi^{+}\right]$, then act on the space of overconvergent modular forms.

We can now state the main result of the paper, namely that an overconvergent modular form, which is an eigenform for the Hecke operators $U_{p, a_{i}}$, can be analytically continued to the whole variety under a certain assumption, and thus is classical. Let $\kappa$ be a weight; explicitly, it is a collection of integers $\left(\kappa_{i, 1} \geq \cdots \geq \kappa_{i, a_{i}}, \lambda_{i, a} \geq \cdots \geq \lambda_{i, b_{i}}\right)_{1 \leq i \leq d}$. Let $S=\left\{a_{1}, \ldots, a_{d}\right\} \cap\left[1, a_{d}+b_{d}-1\right]$. The cardinality of $S$ is exactly the number of canonical subgroups. Let us write $\Sigma_{i}=\left\{j: a_{j}=a_{i}\right\}$ for all $i$.

Theorem 3.17. Let $f$ be an overconvergent modular form of weight $\kappa$. Suppose that $f$ is an eigenform for the Hecke operators $U_{p, a_{i}}$, with eigenvalue $\alpha_{i}$ for $a_{i} \in S$, and that we have the relations

$$
n_{i}+v\left(\alpha_{i}\right)<\inf _{j \in \Sigma_{i}}\left(\kappa_{j, a_{j}}+\lambda_{j, b_{j}}\right)
$$

Then $f$ is classical.

Here $n_{i}$ is a constant depending on the variety. It actually comes from the normalization factor of the Hecke operator $U_{p, a_{i}}$. This theorem is a classicality result, analogous to the one proved by Coleman [1996] in the case of the modular 
curve. Actually, this result has also been proven by Buzzard [2003] and Kassaei [2006] using an analytic continuation method, from which we take inspiration here. Note that there has been extensive work on the classicality problem in the case of the existence of the ordinary locus. We can cite the work of Sasaki [2010], Johansson [2013], Tian and Xiao [2013] and Pilloni and Stroh [2011] in the case of Hilbert varieties, and the work of the author with Pilloni and Stroh [Bijakowski et al. 2016] for more general PEL Shimura varieties (see also [Bijakowski 2014] for Shimura varieties with ramification).

In this introduction, we have assumed that $p$ is inert in $F_{0}$ and splits in $F$. The group associated to the Shimura variety is then a linear group at $p$. If $p$ is inert in $F$, then the group is a unitary group at $p$. Everything we have said adapts to that context: the description of the $\mu$-ordinary locus, the existence of the canonical subgroups, and the analytic continuation theorem. Note that the geometry is more involved in that case; for example, to define Hecke operators, one has to deal with subgroups of $A\left[p^{2}\right]$.

Of course, the assumption that $p$ is inert in $F_{0}$ is for simplicity, so what we have said can be formulated for any prime $p$ unramified in $F$. In the writing of the paper, we have tried to formulate propositions valid in both the linear and unitary cases as much as possible, but of course we often had to treat the proofs separately.

Let us now talk briefly about the text. In Section 1, we introduce the varieties we are dealing with, define the $\mu$-ordinary locus and study the canonical subgroups. In Section 2 we define the classical and overconvergent modular, as well as the Hecke operators. In Section 3 we prove the analytic continuation result. For simplicity, we suppose that the prime $p$ is inert in Sections 2 and 3, and Section 4 briefly shows how to handle the general case.

\section{Shimura varieties of type (A)}

\section{A. The moduli space.}

1A1. Shimura datum. We will introduce the objects needed to define the Shimura variety of unitary type we will work with. We refer to [Kottwitz 1992, Section 5] for more details.

Let $F_{0}$ be a totally real field of degree $d$, and $F$ a CM-extension of $F_{0}$. Let $\left(U_{\mathbb{Q}},\langle\rangle,\right)$ be a nondegenerate hermitian $F$-module and $G$ its automorphism group. For any $\mathbb{Q}$-algebra $R$, we have

$$
G(R)=\left\{(g, c) \in \mathrm{GL}_{F}\left(U_{\mathbb{Q}} \otimes_{\mathbb{Q}} R\right) \times R^{*}:\langle g x, g y\rangle=c\langle x, y\rangle \text { for all } x, y \in U_{\mathbb{Q}} \otimes_{\mathbb{Q}} R\right\} .
$$

Let $\tau_{1}, \ldots, \tau_{d}$ be the embeddings of $F_{0}$ into $\mathbb{R}$, and let $\sigma_{i}$ and $\bar{\sigma}_{i}$ be the two embeddings of $F$ into $\mathbb{C}$ extending $\tau_{i}$. The choice of $\sigma_{i}$ gives an isomorphism $F \otimes_{F_{0}, \tau_{i}} \mathbb{R} \simeq \mathbb{C}$. Let $U_{i}=U_{\mathbb{Q}} \otimes_{F_{0}, \tau_{i}} \mathbb{R}$. We write $\left(a_{i}, b_{i}\right)$ for the signature of the 
antihermitian structure on $U_{i}$. Then $G_{\mathbb{R}}$ is isomorphic to

$$
\mathrm{G}\left(\prod_{i=1}^{d} \mathrm{U}\left(a_{i}, b_{i}\right)\right),
$$

where $a_{i}+b_{i}$ is independent of $i$, and is equal to $(1 / 2 d) \operatorname{dim}_{\mathbb{Q}} U_{\mathbb{Q}}$. We'll call this quantity $a+b$.

We also give ourselves a morphism of $\mathbb{R}$-algebras $h: \mathbb{C} \rightarrow \operatorname{End}_{F} U_{\mathbb{R}}$ such that $\langle h(z) v, w\rangle=\langle v, h(\bar{z}) w\rangle$ and $(v, w) \mapsto\langle v, h(i) w\rangle$ is positive definite. This morphism gives a complex structure on $U_{\mathbb{R}}$; let $U_{\mathbb{C}}^{1,0}$ be the subspace of $U_{\mathbb{C}}$ on which $h(z)$ acts by multiplication by $z$.

We then have $U_{\mathbb{C}}^{1,0} \simeq \prod_{i=1}^{d} \mathbb{C}^{a_{i}} \oplus \overline{\mathbb{C}}^{b_{i}}$ as $F \otimes_{\mathbb{Q}} \mathbb{R}$-modules and $F \otimes_{\mathbb{Q}} \mathbb{R} \simeq \bigoplus_{i=1}^{d} \mathbb{C}$ (where the action of $\mathbb{C}$ on $\mathbb{C}^{a_{i}} \oplus \overline{\mathbb{C}}^{b_{i}}$ is the standard action on the first factor and the conjugated action on the second).

Let $U$ be an $O_{F}$-stable lattice of $U_{\mathbb{Q}}$, and assume that the pairing $\langle$,$\rangle induces a$ pairing $U \times U \rightarrow \mathbb{Z}$ which is perfect at $p$.

The ring $O_{F}$ is a free $\mathbb{Z}$-module. Let $\alpha_{1}, \ldots, \alpha_{t}$ be a basis of this module and

$$
\operatorname{det}_{U^{1,0}}=f\left(X_{1}, \ldots, X_{t}\right)=\operatorname{det}\left(X_{1} \alpha_{1}+\cdots+X_{t} \alpha_{t} ; U_{\mathbb{C}}^{1,0} \otimes_{\mathbb{C}} \mathbb{C}\left[X_{1}, \ldots, X_{t}\right]\right) .
$$

We can show that $f$ is polynomial with algebraic coefficients. The number field $E$ generated by its coefficients is called the reflex field.

Remark 1.1. We chose for simplicity to work with a central algebra. One can easily adapt the arguments here, replacing $F$ by a simple algebra $B$ with center $F$.

1A2. The Shimura variety. Let us now define the PEL Shimura variety of type (A) associated to $G$. Let $K$ be an extension of $\mathbb{Q}_{p}$ containing the images of all the embeddings $F \hookrightarrow \overline{\mathbb{Q}}_{p}$. Assume that $p$ is unramified in $F$. We fix an embedding $E \hookrightarrow K$, so that the coefficients of the polynomial $\operatorname{det}_{U^{1,0}}$ can be seen as elements of $O_{K}$. We also fix an integer $N \geq 3$ prime to $p$.

Definition 1.2. Let $X$ be the moduli space over $O_{K}$ whose $S$-points are the isomorphism classes of $(A, \lambda, \iota, \eta)$, where

- $A \rightarrow S$ is an abelian scheme;

- $\lambda: A \rightarrow A^{t}$ is a prime-to- $p$ polarization;

- เ : $O_{F} \rightarrow$ End $A$ is compatible with complex conjugation and the Rosati involution, and the polynomials $\operatorname{det}_{U^{1,0}}$ and $\operatorname{det}_{\operatorname{Lie}(A)}$ are equal;

- $\eta: A[N] \rightarrow U / N U$ is an $O_{F}$-linear symplectic similitude, which lifts locally for the étale topology to an $O_{F}$-linear symplectic similitude

$$
H_{1}\left(A, \mathbb{A}_{f}^{p}\right) \rightarrow U \otimes_{\mathbb{Z}} \mathbb{A}_{f}^{p}
$$


The moduli space $X$ is representable by a quasiprojective scheme over $O_{K}$. We will make a slight assumption on the variety we consider.

Hypothesis 1.3. We suppose that we are not in the case $d=1$ and $(a, b)=(1,1)$.

This condition is technical, and will ensure that we can neglect the cusps in the definition of the modular forms. The case we exclude corresponds essentially to the modular curve, which is well known.

1A3. Iwahori level. Let $\pi$ be a prime of $F_{0}$ above $p$; we will call $f$ the residual degree and write $q:=p^{f}$. Since $p$ is unramified in $F$, we have two possibilities for the behavior of $p$ in $F$ :

- $\pi$ splits as $\pi^{+} \pi^{-}$in $F$. We say that $\pi$ is in case (L).

- $\pi$ is inert in $F$. We say that $\pi$ is in case (U).

The terminology (L) and (U) comes from the fact that the group $G$ at $\pi$ is a linear or a unitary group, respectively. To define the Iwahori structure, we will break into the two cases.

Definition 1.4. Let $X_{\mathrm{Iw}, \pi}$ be the moduli space of isomorphism classes of tuples $\left(A, \lambda, \iota, \eta, H_{\bullet}\right)$, where

- $(A, \lambda, \iota, \eta)$ is a point of $X$;

- $0 \subset H_{1} \subset \cdots \subset H_{a+b}=A\left[\pi^{+}\right]$, where each $H_{i}$ is a subgroup of $A\left[\pi^{+}\right]$which is locally isomorphic for the étale topology to $\left(O_{F} / \pi^{+}\right)^{i}$ in the case (L);

- $0 \subset H_{1} \subset \cdots \subset H_{a+b}=A[\pi]$, where each $H_{i}$ is a subgroup of $A[\pi]$ which is locally isomorphic for the étale topology to $\left(O_{F} / \pi\right)^{i}$ and such that $H_{a+b-i}=$ $H_{i}^{\perp}$ in the case (U).

The moduli spaces $X_{\mathrm{Iw}, \pi}$ are representable by quasiprojective schemes over $O_{K}$. We also define the full Iwahori space by $X_{\mathrm{Iw}}=X_{\mathrm{Iw}, \pi_{1}} \times_{X} X_{\mathrm{Iw}, \pi_{2}} \times_{X} \cdots \times \times_{X} X_{\mathrm{Iw}, \pi_{g}}$, where $\pi_{1}, \ldots, \pi_{g}$ are the primes of $F_{0}$ above $p$ and the maps $X_{\mathrm{Iw}, \pi_{i}} \rightarrow X$ are the natural morphisms corresponding to forgetting the $\left(H_{i}\right)$.

Remark 1.5. In the case (L), the subgroups $A\left[\pi^{+}\right]$and $A\left[\pi^{-}\right]$are Cartier duals. This comes from the compatibility between the complex conjugation and the Rosati involution. Therefore, each of these groups is totally isotropic. A flag $\left(H_{0}\right)$ of $A\left[\pi^{+}\right]$ gives naturally a flag $\left(H_{\bullet}^{\perp}\right)$ of $A\left[\pi^{-}\right]$with $H_{i}^{\perp}=\left(A\left[\pi^{+}\right] / H_{i}\right)^{D} \subset A\left[\pi^{+}\right]^{D}=A\left[\pi^{-}\right]$. Choosing the prime $\pi^{-}$instead of $\pi^{+}$would have given the same definition.

Now we will explicitly describe the determinant condition for the abelian scheme $A$. We are still working with a prime $\pi$ of $F_{0}$ above $p$, and assume it is of type (L). Let $\Sigma_{\pi}$ be the decomposition group at $\pi$, i.e., the elements $\sigma \in \operatorname{Hom}\left(F_{0}, \overline{\mathbb{Q}}_{p}\right)$ such that $\sigma$ sends $\pi$ into the maximal ideal of $\overline{\mathbb{Q}}_{p}$. For every $\sigma \in \Sigma_{\pi}$, there are two embeddings $\sigma^{+}$and $\sigma^{-}$of $F$ into $\overline{\mathbb{Q}}_{p}$ above $\sigma$; the embedding 
$\sigma^{+}$sends $\pi^{+}$into the maximal ideal of $\overline{\mathbb{Q}}_{p}$, and similarly for $\pi^{-}$. To $\sigma$ we have a pair of integers $\left(a_{\sigma}, b_{\sigma}\right)$ and the choice of the embedding $\sigma^{+}$gives an order for the two elements of the pair. Let $A \rightarrow R$ be an abelian scheme over an $O_{K}$-algebra $R$. If we let $\omega_{\pi}:=e^{*} \Omega_{A\left[\pi^{\infty}\right]}^{1}$, then we have $\omega_{\pi}=\omega_{\pi}^{+} \oplus \omega_{\pi}^{-}$, where $\omega_{\pi}^{+}:=e^{*} \Omega_{A\left[\left(\pi^{+}\right)^{\infty}\right]}^{1}$. The determinant condition for $A$ then implies that

$$
\omega_{\pi}^{+}=\bigoplus_{\sigma \in \Sigma_{\pi}} R^{a_{\sigma}}
$$

with $O_{F}$ acting on $R^{a_{\sigma}}$ by $\sigma^{+}$. Similarly, we have

$$
\omega_{\pi}^{-}=\bigoplus_{\sigma \in \Sigma_{\pi}} R^{b_{\sigma}}
$$

with $O_{F}$ acting on $R^{b_{\sigma}}$ by $\sigma^{-}$.

Now suppose that $\pi$ is of type (U). We still denote by $\Sigma_{\pi}$ the decomposition group at $\pi$ of $F_{0}$. For each $\sigma \in \Sigma_{\pi}$, there are two embeddings $\sigma_{1}$ and $\sigma_{2}$ of $F$ above $\sigma$; the choice of $\sigma_{1}$ gives an order for the elements of the pair $\left(a_{\sigma}, b_{\sigma}\right)$. Let $A \rightarrow R$ be an abelian scheme over an $O_{K}$-algebra $R$. If we let $\omega_{\pi}:=e^{*} \Omega_{A\left[\pi^{\infty}\right]}^{1}$, then the determinant condition for $A$ implies

$$
\omega_{\pi}=\bigoplus_{\sigma \in \Sigma_{\pi}} R^{a_{\sigma}} \oplus R^{b_{\sigma}}
$$

where $O_{F}$ acts by $\sigma_{1}$ on $R^{a_{\sigma}}$ and by $\sigma_{2}$ on $R^{b_{\sigma}}$.

1B. The $\mu$-ordinary locus. We will describe in this section the $\mu$-ordinary locus of the Shimura variety. Let us first introduce some notation.

Let $L$ be an unramified extension of $\mathbb{Q}_{p}$ of degree $f_{0}$ and $k$ a field of characteristic $p$ containing the residue field of $L$. Let $D$ be the Galois group of $L$ over $\mathbb{Q}_{p}$; there is an isomorphism $D \simeq \mathbb{Z} / f_{0} \mathbb{Z}$, where 1 is identified with the Frobenius $\sigma$ of $L$. We will write $W(k)$ for the ring of Witt vectors of $k$. Let $\underline{\varepsilon}=\left(\varepsilon_{\tau}\right)_{\tau \in D}$ be a sequence of integers equal to 0 or 1 . We define a Dieudonné module $M_{\underline{\varepsilon}}$ in the following way: it is a free $W(k)$-module of rank $f_{0}$ and, if $\left(e_{\tau}\right)_{\tau \in D}$ is a basis of this module, then the Frobenius and Verschiebung are defined by

$$
F e_{\sigma^{-1} \tau}=p^{\varepsilon_{\tau}} e_{\tau} \quad \text { and } \quad V e_{\tau}=p^{1-\varepsilon_{\tau}} e_{\sigma^{-1} \tau} .
$$

The module $M_{\underline{\varepsilon}}$ is given an action of the ring of integers of $L$, namely $O_{L}$ acts on $W(k) \cdot e_{\tau}$ by $\tau$.

We'll call $\mathrm{BT}_{\underline{\varepsilon}}$ the $p$-divisible group over $k$ corresponding to the Dieudonné module $M_{\underline{\varepsilon}}$, and $H_{\underline{\varepsilon}}$ the $p$-torsion of this $p$-divisible group.

1B1. Linear case. Now we come back to our Shimura variety. Consider first the case (L); we are still considering a place $\pi$ of $F_{0}$ above $\pi$ which splits as $\pi=\pi^{+} \pi^{-}$ 
in $F$. If $k$ is a field containing the residue field of $O_{K}$ and $x=(A, \lambda, \iota, \eta)$ is a $k$-point of $X$, then whether the abelian variety $A$ is $\mu$-ordinary at $\pi$ will depend on the $p$-divisible group $A\left[\left(\pi^{+}\right)^{\infty}\right]$. Recall that this $p$-divisible group has an action of $O_{F, \pi^{+}}$, the completion of $O_{F}$ at $\pi^{+}$; this is an unramified extension of $\mathbb{Q}_{p}$ of degree $f$. If $\Sigma_{\pi}$ denotes, as before, the decomposition group of $\pi$ in $F_{0}$, then there is a bijection between $\Sigma_{\pi}$ and the Galois group of $O_{F, \pi^{+}}$and, for each $\sigma \in \Sigma_{\pi}$, we have a pair of integers $\left(a_{\sigma}, b_{\sigma}\right)$. We put the elements $\left(a_{\sigma}\right)$ in increasing order; we then have $a_{1} \leq a_{2} \leq \cdots \leq a_{f}$. For each integer $0 \leq i \leq f$ we define the sequence $\underline{\varepsilon}_{i}=\left(\varepsilon_{i, j}\right)_{1 \leq j \leq f}$ by $\varepsilon_{i, j}=1$ if $j \geq i+1$ and $\varepsilon_{i, j}=0$ otherwise. We also set by convention $a_{0}=0$ and $a_{f+1}=a+b$.

Definition 1.6. Let $k$ be an algebraically closed field of characteristic $p$, and let $x=(A, \lambda, \iota, \eta)$ be a $k$-point of $X$. Then $x$ is $\mu$-ordinary at $\pi$ if there is an isomorphism

$$
A\left[\left(\pi^{+}\right)^{\infty}\right] \simeq \prod_{i=0}^{f} \mathrm{BT}_{\underline{\varepsilon}_{i}}^{a_{i+1}-a_{i}}
$$

of $p$-divisible groups with $O_{F, \pi^{+}}$action.

Note that the term on the right-hand side is explicitly

$$
\mathrm{BT}_{(1, \ldots, 1)}^{a_{1}} \times \mathrm{BT}_{(0,1, \ldots, 1)}^{a_{2}-a_{1}} \times \cdots \times \mathrm{BT}_{(0, \ldots, 0,1)}^{a_{f}-a_{f-1}} \times \mathrm{BT}_{(0, \ldots, 0)}^{b_{f}}
$$

Let $X_{0}$ denote the special fiber of $X$, and $X_{0}^{\mu-\pi \text {-ord }}$ the $\mu$-ordinary locus at the place $\pi$. We have the following proposition, due to Wedhorn [1999, Theorem 1.6.2]:

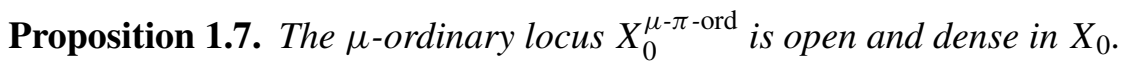

We also have the following characterization of the $\mu$-ordinary locus:

Proposition 1.8 [Moonen 2004, Theorem 1.3.7]. Let $k$ be an algebraically closed field of characteristic $p$, and let $x=(A, \lambda, \iota, \eta)$ be a $k$-point of $X$. Then $x$ is $\mu$-ordinary at $\pi$ if and only if there is an isomorphism

$$
A\left[\pi^{+}\right] \simeq \prod_{i=0}^{f} \mathrm{BT}_{\underline{\varepsilon}_{i}}^{a_{i+1}-a_{i}}\left[\pi^{+}\right]
$$

of finite flat group schemes with $O_{F, \pi^{+}}$action.

Since $\mathrm{BT}_{\underline{\varepsilon_{i}}}$ is multiplicative for $i=0$, étale for $i=f$ and bi-infinitesimal otherwise, we have the following criterion for the existence of the ordinary locus at $\pi$ :

Proposition 1.9. The $\mu$-ordinary locus equals the ordinary locus (at the place $\pi$ ) if and only if there exists an integer a such that $a_{\sigma}=$ a for all $\sigma \in \Sigma_{\pi}$.

This last condition is also equivalent to the fact that the local reflex field at $\pi$ is equal to $\mathbb{Q}_{p}$ (see [Wedhorn 1999, Section 1.6] for more details). 
1B2. Unitary case. Let us now consider the case (U). Let $k$ be a field containing the residue field of $O_{K}$, and let $x=(A, \lambda, \iota, \eta)$ be a $k$-point of $X$. Whether the abelian variety $A$ is $\mu$-ordinary at $\pi$ will depend on the $p$-divisible group $A\left[\pi^{\infty}\right]$. Recall that this $p$-divisible group has an action of $O_{F, \pi}$, the completion of $O_{F}$ at $\pi$; this is an unramified extension of $\mathbb{Q}_{p}$ of degree $2 f$. Recall that $\Sigma_{\pi}$ is the decomposition group at $\pi$ of $F_{0}$ and it is of cardinality $f$. If $\sigma \in \Sigma_{\pi}$, there are two embeddings $\sigma_{1}$ and $\sigma_{2}$ of $F$ into $\overline{\mathbb{Q}}_{p}$, and the choice of one of the two gives elements $a_{\sigma}$ and $b_{\sigma}$. We suppose that the choice is made so that $a_{\sigma} \leq b_{\sigma}$; we also order the elements in $\Sigma_{\pi}$ so that the sequence $\left(a_{\sigma}\right)$ is increasing. We then have

$$
a_{1} \leq a_{2} \leq \cdots \leq a_{f} \leq b_{f} \leq b_{f-1} \leq \cdots \leq b_{1} .
$$

This gives an order on the embeddings of $F$ into $\overline{\mathbb{Q}}_{p}$. For each integer $0 \leq i \leq 2 f$ we define the sequence $\underline{\varepsilon}_{i}=\left(\varepsilon_{i, j}\right)_{1 \leq j \leq 2 f}$ by $\varepsilon_{i, j}=1$ if $j \geq i+1$ and $\varepsilon_{i, j}=0$ otherwise. We define a sequence $\left(\alpha_{i}\right)_{0 \leq i \leq 2 f+1}$ by $\alpha_{0}=0, \alpha_{i}=a_{i}$ for $1 \leq i \leq f$, $\alpha_{i}=b_{2 f+1-i}$ for $f+1 \leq i \leq 2 f$ and $\alpha_{2 f+1}=a+b$.

Definition 1.10. Let $k$ be an algebraically closed field of characteristic $p$, and let $x=(A, \lambda, \iota, \eta)$ be a $k$-point of $X$. Then $x$ is $\mu$-ordinary at $\pi$ if there is an isomorphism

$$
A\left[\pi^{\infty}\right] \simeq \prod_{i=0}^{2 f} \mathrm{BT}_{\underline{\varepsilon}_{i}}^{\alpha_{i+1}-\alpha_{i}}
$$

of $p$-divisible groups with $O_{F, \pi}$ action.

Note that the term in the right-hand side is explicitly

$$
\begin{aligned}
\mathrm{BT}_{(1, \ldots, 1)}^{a_{1}} \times \mathrm{BT}_{(0,1, \ldots, 1)}^{a_{2}-a_{1}} \times \cdots & \times \mathrm{BT}_{(0, \ldots, 0,1,1 \ldots, 1)}^{a_{f}-a_{f-1}} \\
& \times \mathrm{BT}_{(0, \ldots, 0,1 \ldots, 1)}^{b_{f}-a_{f}} \times \mathrm{BT}_{(0, \ldots, 0,0,1 \ldots, 1)}^{a_{f}-a_{f-1}} \times \cdots \times \mathrm{BT}_{(0, \ldots, 0)}^{a_{1}} .
\end{aligned}
$$

(We have used the fact that $b_{i}-b_{i-1}=a_{i-1}-a_{i}$, since the quantity $a_{j}+b_{j}$ is independent of $j$.)

Let $X_{0}$ denote the special fiber of $X$, and $X_{0}^{\mu-\pi \text {-ord }}$ the $\mu$-ordinary locus. We have the following proposition, due to Wedhorn [1999, Theorem 1.6.2]:

Proposition 1.11. The $\mu$-ordinary locus $X_{0}^{\mu-\pi \text {-ord }}$ is open and dense in $X_{0}$.

We also have the following characterization of the $\mu$-ordinary locus:

Proposition 1.12 [Moonen 2004, Theorem 1.3.7]. Let $k$ be an algebraically closed field of characteristic $p$, and let $x=(A, \lambda, \iota, \eta)$ be a k-point of $X$. Then $x$ is $\mu$-ordinary at $\pi$ if and only if there is an isomorphism

$$
A[\pi] \simeq \prod_{i=0}^{2 f} \mathrm{BT}_{\underline{\varepsilon}_{i}}^{\alpha_{i+1}-\alpha_{i}}[\pi]
$$

of finite flat group schemes with $O_{F, \pi}$ action. 
Since $\mathrm{BT}_{\underline{\varepsilon}_{i}}$ is multiplicative for $i=0$, étale for $i=2 f$ and bi-infinitesimal otherwise, we have the following criterion for the existence of the ordinary locus at $\pi$ :

Proposition 1.13. The $\mu$-ordinary locus equals the ordinary locus (at the place $\pi$ ) if and only if $a_{\sigma}=b_{\sigma}=\frac{1}{2}(a+b)$ for all $\sigma \in \Sigma_{\pi}$.

Again, this last condition is equivalent to the fact that the local reflex field at $\pi$ is equal to $\mathbb{Q}_{p}$.

We'll need later to work with the rigid space associated to $X$. Let us call this rigid space $X_{\text {rig }}$; it is the generic fiber of the formal completion of $X$ along its special fiber. We refer to [Berthelot 1996] for more details on rigid spaces. We have a specialization map sp : $X_{\text {rig }} \rightarrow X_{0}$, and we'll write $X_{\text {rig }}^{\mu \text {-ord }}$ for the inverse image of the $\mu$-ordinary locus under the specialization map. We'll also write $X_{\text {Iw,rig }}$ for the rigid space associated to $X_{\text {Iw }}$.

\section{C. Canonical subgroups.}

1C1. Degrees and partial degrees. Before introducing the canonical subgroups on the $\mu$-ordinary locus, we'll define the degree for a finite flat group scheme defined over a finite extension of $\mathbb{Q}_{p}$, and the partial degrees for these endowed with an action of the ring of integers of an unramified extension of $\mathbb{Q}_{p}$.

Definition 1.14. Let $L$ be a finite extension of $\mathbb{Q}_{p}$ and let $G$ be a finite flat group scheme of $p$-power order over $O_{L}$. Let $\omega_{G}$ be the conormal module along the unit section. Then the degree of $G$ is, by definition,

$$
\operatorname{deg} G:=v\left(\operatorname{Fitt}_{0} \omega_{G}\right),
$$

where Fitt ${ }_{0}$ denotes the Fitting ideal, and the valuation of an ideal is defined by $v\left(x O_{L}\right)=v(x)$, normalized by $v(p)=1$.

We now state some properties of this function. We refer to [Fargues 2010, Section 3] for more details.

Proposition 1.15. The degree function has the following properties:

- Let $G$ be as before. Then, if $G^{D}$ denotes the Cartier dual of $G$, we have

$$
\operatorname{deg} G^{D}=\operatorname{ht} G-\operatorname{deg} G .
$$

In particular, $\operatorname{deg} G \in[0$, ht $G]$.

- The degree function is additive: if we have an exact sequence

$$
0 \rightarrow G_{1} \rightarrow G_{2} \rightarrow G_{3} \rightarrow 0
$$

with $G_{i}$ finite flat, then $\operatorname{deg} G_{2}=\operatorname{deg} G_{1}+\operatorname{deg} G_{3}$. 
- Let $G$ and $G^{\prime}$ be two finite flat group schemes, and suppose that there exists a morphism $f: G \rightarrow G^{\prime}$ which is an isomorphism in generic fiber. Then $\operatorname{deg} G \leq \operatorname{deg} G^{\prime}$, and we have equality if and only if $f$ is an isomorphism.

We deduce from the last property the following corollary:

Corollary 1.16. Let $G$ be a finite flat group scheme of p-power order defined over a finite extension of $\mathbb{Q}_{p}$. Suppose that $H_{1}$ and $H_{2}$ are two finite flat subgroups of $G$. Then

$$
\operatorname{deg} H_{1}+\operatorname{deg} H_{2} \leq \operatorname{deg}\left(H_{1}+H_{2}\right)+\operatorname{deg}\left(H_{1} \cap H_{2}\right) .
$$

Proof. By dividing everything by $H_{1} \cap H_{2}$, we are reduced to the case $H_{1} \cap H_{2}=\{0\}$. The morphism $H_{1} \times H_{2} \rightarrow H_{1}+H_{2}$ is an isomorphism in generic fiber, thus by the previous proposition we get

$$
\operatorname{deg}\left(H_{1} \times H_{2}\right) \leq \operatorname{deg}\left(H_{1}+H_{2}\right) .
$$

But, since the degree function is additive, $\operatorname{deg}\left(H_{1} \times H_{2}\right)=\operatorname{deg} H_{1}+\operatorname{deg} H_{2}$.

Let $G$ be as in the previous definition and suppose now that $G$ has an action of $O_{M}$, where $M$ is a finite unramified extension of $\mathbb{Q}_{p}$. Let $\Sigma$ be the set of embeddings of $M$ into $\overline{\mathbb{Q}}_{p}$. Then the module $\omega_{G}$ has an action of $O_{M}$ and has the decomposition

$$
\omega_{G}=\bigoplus_{\sigma \in \Sigma} \omega_{G, \sigma}
$$

where $O_{M}$ acts on $\omega_{G, \sigma}$ by $\sigma$.

Definition 1.17. The partial degree of $G$ is defined for all $\sigma \in \Sigma$ as

$$
\operatorname{deg}_{\sigma} G=v\left(\operatorname{Fitt}_{0} \omega_{G, \sigma}\right) .
$$

Proposition 1.18. The partial degree functions have the following properties:

- We have $\operatorname{deg} G=\sum_{\sigma \in \Sigma} \operatorname{deg}_{\sigma} G$.

- Suppose that $G$ has height $\left[M: \mathbb{Q}_{p}\right]$. If $G^{D}$ denotes the Cartier dual of $G$, we have, for all $\sigma \in \Sigma$,

$$
\operatorname{deg}_{\sigma} G^{D}=h-\operatorname{deg}_{\sigma} G .
$$

In particular, $\operatorname{deg}_{\sigma} G \in[0, h]$.

- The partial degree functions are additive: if we have an exact sequence

$$
0 \rightarrow G_{1} \rightarrow G_{2} \rightarrow G_{3} \rightarrow 0
$$

with $G_{i}$ finite flat with an action of $O_{M}$, then $\operatorname{deg}_{\sigma} G_{2}=\operatorname{deg}_{\sigma} G_{1}+\operatorname{deg}_{\sigma} G_{3}$ for all $\sigma \in \Sigma$.

We refer to [Bijakowski 2014, Section 1.1.5] for more details. 
Remark 1.19. If we are in the situation of the third point of Proposition 1.15, i.e., if we have a morphism of finite flat group schemes $f: G \rightarrow G^{\prime}$ that is an isomorphism in generic fiber and if $G$ and $G^{\prime}$ have an action of $O_{M}$, then it is not true that the partial degrees increase. Indeed, the functions that increase are linear combinations of the partial degrees. For example, if $\left[M: \mathbb{Q}_{p}\right]=2$, there are two partial degrees $\operatorname{deg}_{1}$ and $\operatorname{deg}_{2}$, and the functions that increase are $\operatorname{deg}_{1}+p \operatorname{deg}_{2}$ and $p \operatorname{deg}_{1}+\operatorname{deg}_{2}$. See [Bijakowski 2014, Proposition 1.1.33] for more details.

Example 1.20. Let us apply what we have said to our Shimura variety. Let $x=$ $(A, \lambda, \iota, \eta)$ be an $O_{L}$-point of $X$ (where $L$ is a finite extension of $\mathbb{Q}_{p}$ ) and suppose that $\pi$ is a place of $F_{0}$ above $p$ in case (L). Then $A\left[\pi^{+}\right]$has an action of $O_{F_{0}, \pi}$. Moreover, we have

$$
\operatorname{deg}_{\sigma} A\left[\pi^{+}\right]=a_{\sigma}
$$

for all $\sigma \in \Sigma_{\pi}$. If $H$ is an $O_{F}$-stable subgroup of $A\left[\pi^{+}\right]$of height $f h$, then the orthogonal $H^{\perp}$ is a subgroup of $A\left[\pi^{-}\right]$of height $f(a+b-h)$. We have $H^{\perp} \simeq\left(A\left[\pi^{+}\right] / H\right)^{D}$, and thus

$$
\operatorname{deg}_{\sigma} H^{\perp}=(a+b-h)-\left(a_{\sigma}-\operatorname{deg}_{\sigma} H\right)=b_{\sigma}-h+\operatorname{deg}_{\sigma} H
$$

for all $\sigma \in \Sigma_{\pi}$. We see that one has the inequalities

$$
\operatorname{deg}_{\sigma} H \geq h-b_{\sigma} \quad \text { and } \quad \operatorname{deg}_{\sigma} H^{\perp} \geq b_{\sigma}-h
$$

for all $\sigma \in \Sigma_{\pi}$.

Example 1.21. Suppose now that $\pi$ is in case (U). Then the group scheme $A[\pi]$ has an action of $O_{F, \pi}$. Recall that if $\sigma \in \Sigma_{\pi}$ is an embedding of $F_{0}$ into $\overline{\mathbb{Q}}_{p}$ above $\pi$, then there are two embeddings $\sigma_{1}$ and $\sigma_{2}$ of $F$ extending $\sigma$. With our previous conventions, we have

$$
\operatorname{deg}_{\sigma_{1}} A[\pi]=a_{\sigma} \quad \text { and } \quad \operatorname{deg}_{\sigma_{2}} A[\pi]=b_{\sigma} .
$$

If $H$ is an $O_{F}$-stable subgroup of $A[\pi]$ of height $2 f h$, then the orthogonal $H^{\perp}$ is a subgroup of $A[\pi]$ of height $2 f(a+b-h)$. We have $H^{\perp} \simeq(A[\pi] / H)^{D, c}$, where the superscript $c$ means that the action of $O_{F}$ on $(A[\pi] / H)^{D, c}$ is the conjugate of the natural one. This comes from the compatibility between the Rosati involution and the complex conjugation. Thus,

$$
\operatorname{deg}_{\sigma_{1}} H^{\perp}=a_{\sigma}-h+\operatorname{deg}_{\sigma_{2}} H \quad \text { and } \quad \operatorname{deg}_{\sigma_{2}} H^{\perp}=b_{\sigma}-h+\operatorname{deg}_{\sigma_{1}} H
$$

for all $\sigma \in \Sigma_{\pi}$. We see that one has the inequalities

$$
\operatorname{deg}_{\sigma_{1}} H \geq h-b_{\sigma} \quad \text { and } \quad \operatorname{deg}_{\sigma_{2}} H \geq h-a_{\sigma}
$$

for all $\sigma \in \Sigma_{\pi}$. 
1C2. Siegel variety. Let us now recall some facts for the canonical subgroup of the Siegel variety.

Let $g \geq 1$ be an integer and $\mathcal{A}_{g}$ the Siegel variety. There is a universal abelian scheme $A$ on $\mathcal{A}_{g}$. There is also a Hasse invariant $\mathrm{Ha}$ on $\mathcal{A}_{g}$. We quote the main result obtained by Fargues [2011] on the canonical subgroup.

Proposition 1.22. Let $A$ be an abelian scheme of dimension $g$ defined over $O_{L}$ ( $L$ is a finite extension of $\mathbb{Q}_{p}$ ). Suppose that the valuation $w$ of the Hasse invariant is strictly less than $\frac{1}{2}$. Then there is a canonical subgroup $H \subset A[p]$, of height $g$, totally isotropic, with

$$
\operatorname{deg} H=g-w \text {. }
$$

Let $\mathcal{A}_{g \text {,rig }}$ be the rigid space associated to $\mathcal{A}_{g}$. Then the ordinary locus of $\mathcal{A}_{g}$,rig is defined as the locus where the associated abelian scheme is ordinary; it is also the locus where the Hasse invariant is invertible. The proposition says that, on a strict neighborhood of the ordinary locus, there exists a canonical subgroup of high degree in $A[p]$. We propose a simple reformulation of this property. We have the following observation:

Proposition 1.23. Let $A$ be an abelian scheme of dimension $g$ defined over $O_{L}$ ( $L$ is a finite extension of $\mathbb{Q}_{p}$ ). There exists at most one subgroup $H$ of height $g$ of $A[p]$ with

$$
\operatorname{deg} H>g-\frac{1}{2} .
$$

Proof. Suppose not, and let $H_{1}$ and $H_{2}$ be two subgroups with deg $H_{i}>g-\frac{1}{2}$. Then we have

$$
2 g-1<\operatorname{deg} H_{1}+\operatorname{deg} H_{2} \leq \operatorname{deg}\left(H_{1}+H_{2}\right)+\operatorname{deg}\left(H_{1} \cap H_{2}\right) .
$$

But $\operatorname{deg}\left(H_{1}+H_{2}\right) \leq \operatorname{deg} A[p]=g$ and, since $H_{1} \cap H_{2}$ is of height $h \leq g-1$, we have $\operatorname{deg}\left(H_{1} \cap H_{2}\right) \leq g-1$. We get a contradiction.

This can be used to prove the existence of the canonical subgroup in the following way. Let $\mathcal{A}_{g}^{\prime}$ be the Siegel variety parametrizing a $g$-dimensional abelian scheme with polarization and a subgroup $H$ totally isotropic of height $g$. We have a map $f: \mathcal{A}_{g}^{\prime} \rightarrow \mathcal{A}_{g}$ corresponding to forgetting $H$. If we let $\mathcal{A}_{g \text {,rig }}^{\prime}$ be the rigid space associated to $\mathcal{A}_{g}^{\prime}$, we still have a morphism $f: \mathcal{A}_{g \text {,rig }}^{\prime} \rightarrow \mathcal{A}_{g \text {,rig. Define }}$ $X_{r}=\left\{x \in \mathcal{A}_{g, \text { rig }}^{\prime}: \operatorname{deg} H(x) \geq g-r\right\}$ for any rational $r$; it is an admissible open subset of $\mathcal{A}_{g \text {,rig }}^{\prime}$. Then the ordinary locus of $\mathcal{A}_{g \text {,rig }}$ is $f\left(X_{0}\right)$, and it follows from [Bijakowski et al. 2016, Proposition 4.1.7] that $\left(f\left(X_{r}\right)\right)_{r>0}$ forms a basis of strict neighborhoods of the ordinary locus (the map $f$ is finite étale, and the $\left(X_{r}\right)_{r>0}$ are strict neighborhoods of $\left.X_{0}\right)$. The previous proposition shows that on $f\left(X_{r}\right)$ there is exactly one subgroup of height $g$ of degree greater than or equal to $g-r$ for $r<\frac{1}{2}$; this is the canonical subgroup. 
1C3. Linear case. Now let's get back to our Shimura varieties. Suppose we are in case $(\mathrm{L})$. Then we have the following proposition:

Proposition 1.24. Let $L$ be a finite extension of $\mathbb{Q}_{p}$ and $x=(A, \lambda, \iota, \eta)$ an $O_{L^{-}}$ point of $X$. Let $1 \leq i \leq f$ be an integer. Then there exists at most one subgroup $H \subset A\left[\pi^{+}\right]$that is stable by $O_{F}$ of height $f a_{i}$ such that

$$
\operatorname{deg} H>\sum_{j=1}^{f} \min \left(a_{j}, a_{i}\right)-\frac{1}{2}
$$

Proof. Suppose not, and let $H_{1}$ and $H_{2}$ be two such subgroups. Let us denote by $f h$ the height of $H_{1} \cap H_{2}$; the height of $H_{1}+H_{2}$ is then $f\left(2 a_{i}-h\right)$. We have

$$
\operatorname{deg} H_{1}+\operatorname{deg} H_{2} \leq \operatorname{deg}\left(H_{1}+H_{2}\right)+\operatorname{deg}\left(H_{1} \cap H_{2}\right) \text {. }
$$

But

$$
\operatorname{deg}\left(H_{1}+H_{2}\right) \leq \sum_{j=1}^{f} \min \left(a_{j}, 2 a_{i}-h\right) \leq \sum_{j=1}^{i} a_{j}+\sum_{j=i+1}^{f}\left(2 a_{i}-h\right)
$$

and

$$
\operatorname{deg}\left(H_{1} \cap H_{2}\right) \leq \sum_{j=1}^{f} \min \left(a_{j}, h\right) \leq \sum_{j=1}^{i-1} a_{j}+\sum_{j=i}^{f} h .
$$

We finally get

$$
\operatorname{deg} H_{1}+\operatorname{deg} H_{2} \leq 2 \sum_{j=1}^{i-1} a_{j}+2 \sum_{j=i+1}^{f} a_{i}+a_{i}+h \leq 2 \sum_{j=1}^{f} \min \left(a_{j}, a_{i}\right)-1
$$

since $h \leq a_{i}-1$, a contradiction.

The proposition shows that there exists at most one subgroup of height $f a_{i}$ and of big degree. The next proposition shows that if two such subgroups exist (with different heights), then we automatically have an inclusion.

Proposition 1.25. Let $i<j$ be two integers between 1 and $f$. Let $x=(A, \lambda, \iota, \eta)$ be an $O_{L}$-point of $X$, and suppose there exists, for $l \in\{i, j\}$, a subgroup $H_{l} \subset A\left[\pi^{+}\right]$ that is stable by $O_{F}$ of height $f a_{l}$ such that

$$
\operatorname{deg} H_{l}>\sum_{k=1}^{f} \min \left(a_{k}, a_{l}\right)-\frac{1}{2}
$$

Then we have $H_{i} \subset H_{j}$. 
Proof. Let $f h$ denote the height of $H_{i} \cap H_{j}$. We have the following inequalities:

$$
\begin{aligned}
& \operatorname{deg}\left(H_{i}+H_{j}\right) \leq \sum_{k=1}^{f} \min \left(a_{k}, a_{i}+a_{j}-h\right) \leq \sum_{k=1}^{j} a_{k}+\sum_{k=j+1}^{f}\left(a_{i}+a_{j}-h\right), \\
& \operatorname{deg}\left(H_{i} \cap H_{j}\right) \leq \sum_{k=1}^{f} \min \left(a_{k}, h\right) \leq \sum_{k=1}^{i-1} a_{k}+\sum_{k=i}^{f} h .
\end{aligned}
$$

We then get

$$
\begin{aligned}
\operatorname{deg} H_{i}+\operatorname{deg} H_{j} & \leq \operatorname{deg}\left(H_{i}+H_{j}\right)+\operatorname{deg}\left(H_{i} \cap H_{j}\right) \\
& \leq 2 \sum_{k=1}^{i-1} a_{k}+\sum_{k=i}^{j}\left(a_{k}+h\right)+\sum_{k=j+1}^{f}\left(a_{i}+a_{j}\right) .
\end{aligned}
$$

If we do not have the inclusion $H_{i} \subset H_{j}$, then $h \leq a_{i}-1$. We then get

$$
\operatorname{deg} H_{i}+\operatorname{deg} H_{j} \leq \sum_{k=1}^{f}\left(\min \left(a_{k}, a_{i}\right)+\min \left(a_{k}, a_{j}\right)\right)-(j-i+1) .
$$

We get a contradiction with the hypothesis that $H_{i}$ and $H_{j}$ have big degrees.

As a consequence, we directly get the existence of canonical subgroups for $\mu$-ordinary abelian schemes. Let $s$ be the cardinality of $\left\{a_{1}, \ldots, a_{f}\right\} \cap[1, a+b-1]$, and denote by $A_{1}<\cdots<A_{s}$ the different elements of this set.

Corollary 1.26. Let $L$ be a finite extension of $\mathbb{Q}_{p}$, and let $x=(A, \lambda, \iota, \eta)$ be an $O_{L^{-}}$ point of $X$. Assume that $x$ is $\mu$-ordinary (i.e., the special fiber of $A$ is $\mu$-ordinary). Then, for any integer $1 \leq k \leq s$, there exists a unique subgroup $H_{k} \subset A\left[\pi^{+}\right]$of height $f A_{k}$, with

$$
\operatorname{deg}_{\sigma} H_{k}=\min \left(a_{\sigma}, A_{k}\right)
$$

for all $\sigma \in \Sigma_{\pi}$.

Proof. We can work over $\overline{\mathbb{Q}}_{p}$. Since $A$ is $\mu$-ordinary we have, by [Moonen 2004, Proposition 2.1.9], a filtration

$$
X_{0}=0 \subset X_{1} \subset \cdots \subset X_{f+1}=A\left[\left(\pi^{+}\right)^{\infty}\right]
$$

on $A\left[\left(\pi^{+}\right)^{\infty}\right]$ with $X_{i} p$-divisible groups such that $\left(X_{i+1} / X_{i}\right) \times \overline{\mathbb{F}}_{p} \simeq \mathrm{BT}_{\underline{\varepsilon}_{i}}^{a_{i+1}-a_{i}}$ for $0 \leq i \leq f$. Let $Y_{i}=X_{i+1} / X_{i}$ for $0 \leq i \leq f$. Then $Y_{i}$ is a $p$-divisible group over the ring of integers of $\overline{\mathbb{Q}}_{p}$. The module $\omega_{Y_{i}}$ decomposes into $\bigoplus_{\sigma \in \Sigma_{\pi}} \omega_{Y_{i}, \sigma}$, and each $\omega_{Y_{i}, \sigma}$ is free over the ring of integers of $\overline{\mathbb{Q}}_{p}$. Recall that we have chosen an ordering for the set $\Sigma_{\pi}=\left\{\sigma_{1}, \ldots, \sigma_{f}\right\}$. From the description of the special fiber of $Y_{i}$, one sees that $\omega_{Y_{i}, \sigma_{j}}$ is 0 if $j \leq i$, and is free of rank $a_{i+1}-a_{i}$ over the ring of integers of $\overline{\mathbb{Q}}_{p}$ if $j \geq i+1$. Thus, $\operatorname{deg}_{\sigma_{j}} Y_{i}\left[\pi^{+}\right]$is 0 if $j \leq i$ and $a_{i+1}-a_{i}$ otherwise. 
Let $H_{i}=X_{i}\left[\pi^{+}\right]$for $0 \leq i \leq f$; it is a finite flat subgroup of $A\left[\pi^{+}\right]$of height $f a_{i}$. Moreover, we have

$$
\operatorname{deg}_{\sigma_{j}} H_{i}=\sum_{k=1}^{i} \operatorname{deg}_{\sigma_{j}} H_{k} / H_{k-1}=\sum_{k=1}^{\min (i, j)}\left(a_{k}-a_{k-1}\right)=a_{\min (i, j)}=\min \left(a_{i}, a_{j}\right)
$$

for all $i$ and $j$ between 1 and $f$. This gives the existence of the desired subgroups. The uniqueness follows from the previous proposition.

Remark 1.27. We also have the following description of the canonical subgroups in the special fiber. Let $L$ be a finite extension of $\mathbb{Q}_{p}$, and let $x=(A, \lambda, \iota, \eta)$ be a $\mu$-ordinary point of $X$ defined over $O_{L}$. Let $A_{s}$ denote the special fiber of $A$; then the Frobenius $F$ acts on $A_{s}\left[\left(\pi^{+}\right)^{\infty}\right]$, and we can form the subgroups

$$
C_{i}:=\left(\pi^{+}\right)^{f-i} A_{s}\left[F^{f},\left(\pi^{+}\right)^{f-i+1}\right]
$$

for $1 \leq i \leq f$. Then we have $0 \subset C_{1} \subset \cdots \subset C_{f} \subset A_{s}\left[\pi^{+}\right]$, and the special fiber of each of the canonical subgroups is equal to one of the $C_{i}$. More precisely, we have $H_{j} \times k(L)=C_{r(j)}$ for $1 \leq j \leq s$, with $k(L)$ the residue field of $L$ and $r(j)=\min \left\{l: a_{l}=A_{j}\right\}$.

We can then define the relevant degree functions on $X_{\mathrm{Iw}, \pi}$. For each integer $j$, define

$$
d_{j}=\sum_{\sigma \in \Sigma_{\pi}} \min \left(a_{\sigma}, a_{j}\right)
$$

Let $X_{\mathrm{Iw}, \pi, \text { rig }}$ be the associated rigid space. We define the degree function Deg : $X_{\mathrm{Iw}, \pi, \text { rig }} \rightarrow \prod_{j=1}^{s}\left[0, d_{j}\right]$ on this space by

$$
\operatorname{Deg}\left(A, \lambda, \iota, \eta, H_{\bullet}\right):=\left(\operatorname{deg} H_{a_{j}}\right)_{1 \leq j \leq s} .
$$

We also define the $j$-th degree function by $\operatorname{Deg}_{j}\left(A, \lambda, \iota, \eta, H_{\bullet}\right):=\operatorname{deg} H_{a_{j}}$ for $1 \leq j \leq s$.

Remark 1.28. The integers $s$ and $d_{j}$ as well as the functions Deg and Deg ${ }_{j}$ depend on the place $\pi$. If the context is clear, we choose not to write the dependence on $\pi$ to lighten the notation.

We then have the following description of the $\mu$-ordinary locus.

Proposition 1.29. The space $X_{\text {rig }}^{\mu-\pi \text {-ord }} \subset X_{\text {rig }}$ is exactly the image of

$$
\operatorname{Deg}^{-1}\left(\left\{d_{1}\right\} \times \cdots \times\left\{d_{s}\right\}\right)
$$

under the map $X_{\mathrm{Iw}, \pi, \text { rig }} \rightarrow X_{\text {rig. }}$. 
Proof. If $x$ is a $\mu$-ordinary point, it then follows from the previous corollary that there exist subgroups $H_{j}$ of height $f a_{j}$ with $\operatorname{deg} H_{j}=d_{j}$ for all $1 \leq j \leq s$. Conversely, suppose that $(A, \lambda, \iota, \eta)$ is a point of $X_{\text {rig }}$, with $A$ defined over the ring of integers of an extension $L$ of $\mathbb{Q}_{p}$, and that there exist subgroups $H_{j}$ of height $f a_{j}$ with $\operatorname{deg} H_{j}=d_{j}$ for all $1 \leq j \leq s$. We want to show that $A$ is $\mu$-ordinary; it suffices to show that $A\left[\pi^{+}\right]$has a nice description. We have $\operatorname{deg}_{\sigma} H_{j} \leq \min \left(a_{\sigma}, a_{j}\right)$ for all $\sigma \in \Sigma_{\pi}$ and, since $d_{j}=\sum_{\sigma \in \Sigma_{\pi}} \min \left(a_{\sigma}, a_{j}\right)$, this forces the last inequality to be an equality. Define $H_{0}=0, H_{s+1}=A\left[\pi^{+}\right]$, and let $H_{j}^{\prime}$ be a complement of $H_{j-1}$ in $H_{j}$ for all $1 \leq j \leq s+1$. This is possible if the field $L$ is large enough. We claim that, for $1 \leq j \leq s+1$,

$$
H_{j} \simeq H_{j}^{\prime} \times H_{j-1} .
$$

Indeed we have a morphism $H_{j-1} \rightarrow H_{j} / H_{j}^{\prime}$ that is an isomorphism in generic fiber. The degree of the image of $H_{j-1}$ in $H_{j} / H_{j}^{\prime}$ thus increases, but since the degree of $H_{j-1}$ is maximal, it must be an equality. We deduce that deg $H_{j}=\operatorname{deg} H_{j}^{\prime}+\operatorname{deg} H_{j-1}$ and that the morphism $H_{j}^{\prime} \times H_{j-1} \rightarrow H_{j}$ is an isomorphism.

We finally get

$$
A\left[\pi^{+}\right] \simeq H_{1}^{\prime} \times H_{2}^{\prime} \times \cdots \times H_{s+1}^{\prime} .
$$

But we can explicitly describe the groups $H_{j}^{\prime}$. Indeed, for all $1 \leq j \leq s+1$ and $\sigma \in \Sigma_{\pi}$, we have (setting $A_{0}=0$ and $A_{s+1}=a+b$ )

$$
\operatorname{deg}_{\sigma} H_{j}^{\prime}=\operatorname{deg}_{\sigma} H_{j}-\operatorname{deg}_{\sigma} H_{j-1}=\min \left(a_{\sigma}, a_{j}\right)-\min \left(a_{\sigma}, A_{j-1}\right) .
$$

This quantity is 0 if $a_{\sigma} \leq A_{j-1}$ and $a_{j}-A_{j-1}$ if $a_{\sigma} \geq a_{j}$. Since the height of $H_{j}^{\prime}$ is $f\left(a_{j}-A_{j-1}\right)$, one can see that the special fiber of $H_{j}^{\prime}$ is isomorphic to $\mathrm{BT}_{\underline{\varepsilon}_{s(j)}}\left[\pi^{+}\right]^{A_{j}-A_{j-1}}$, where $s(j)$ is the number of $\sigma \in D_{\pi}$ with $a_{\sigma} \leq A_{j-1}$ (one can see this by looking at the Dieudonné module associated to the special fiber of $H_{j}^{\prime}$, for example). We then conclude that $A$ is $\mu$-ordinary by Proposition 1.8.

1C4. Unitary case. Suppose now we are in case (U). Then we have the following proposition:

Proposition 1.30. Let $L$ be a finite extension of $\mathbb{Q}_{p}$ and $x=(A, \lambda, \iota, \eta)$ an $O_{L^{-}}$ point of $X$. Let $1 \leq i \leq f$ be an integer. Then there exists at most one subgroup $H \subset A[\pi]$ that is stable by $O_{F}$ of height $2 f a_{i}$ such that

$$
\operatorname{deg} H>\sum_{j=1}^{2 f} \min \left(\alpha_{j}, a_{i}\right)-\frac{1}{2} .
$$

Moreover, if such a subgroup exists, it is totally isotropic.

Proof. The proof of the first part of the proposition is exactly the same as in the linear case (see Proposition 1.24). To prove that the subgroup is totally isotropic, 
we will use the same argument as in the proof of Proposition 1.25. We only need to get a bound for the degree of $H^{\perp}$. But we have $H^{\perp} \simeq(A[\pi] / H)^{D}$, so

$$
\begin{aligned}
\operatorname{deg} H^{\perp} & =2 f b_{i}-\operatorname{deg}(A[\pi] / H) \\
& =\operatorname{deg} H+\sum_{j=1}^{2 f}\left(b_{i}-\alpha_{j}\right)>\sum_{j=1}^{2 f}\left(b_{i}-\alpha_{j}+\min \left(\alpha_{j}, a_{i}\right)\right)-\frac{1}{2} .
\end{aligned}
$$

But $b_{i}-\alpha_{j}+\min \left(\alpha_{j}, a_{i}\right)=\min \left(b_{i}, a_{i}+b_{i}-\alpha_{j}\right)$ and, since $a_{i}+b_{i}$ is constant, we have $a_{i}+b_{i}-\alpha_{j}=\alpha_{2 f+1-i}$. In conclusion, we get

$$
\operatorname{deg} H^{\perp}>\sum_{j=1}^{2 f} \min \left(b_{i}, \alpha_{j}\right)-\frac{1}{2} .
$$

We conclude that $H \subset H^{\perp}$ by applying the proof of Proposition 1.25 directly (note that $H^{\perp}$ is of height $\left.2 f b_{i}\right)$.

The proposition shows that there exists at most one subgroup of height $2 f a_{i}$ and of big degree. The next proposition shows that if two such subgroups exists (with different heights), then we automatically have an inclusion.

Proposition 1.31. Let $i<j$ be two integers between 1 and $f$. Let $x=(A, \lambda, \iota, \eta)$ be an $O_{L}$-point of $X$, and suppose there exists, for $l \in\{i, j\}$, a subgroup $H_{l} \subset A[\pi]$ that is stable by $O_{F}$ of height $2 f a_{l}$ such that

$$
\operatorname{deg} H_{l}>\sum_{k=1}^{2 f} \min \left(\alpha_{k}, a_{l}\right)-\frac{1}{2} .
$$

Then $H_{i} \subset H_{j}$.

Proof. The proof is the same as in the linear case (see Proposition 1.25).

As a consequence, we directly get the existence of canonical subgroups for $\mu$-ordinary abelian schemes. Let $s$ be the cardinality of $\left\{a_{1}, \ldots, a_{f}\right\} \cap\left[1, \frac{1}{2}(a+b)\right]$, and denote by $A_{1}<\cdots<A_{s}$ the different elements of this set.

Corollary 1.32. Let $L$ be a finite extension of $\mathbb{Q}_{p}$ and let $x=(A, \lambda, \iota, \eta)$ be an $O_{L^{-}}$ point of $X$. Assume that $x$ is $\mu$-ordinary (i.e., the special fiber of $A$ is $\mu$-ordinary). Then, for any integer $1 \leq k \leq s$, there exists a unique totally isotropic subgroup $H_{k} \subset A[\pi]$ of height $2 f A_{k}$, with

$$
\operatorname{deg}_{\sigma} H_{k}=\min \left(\alpha_{\sigma}, A_{k}\right)
$$

for all $\sigma \in \Sigma_{\pi}$.

Proof. The proof is similar to the linear case (see Corollary 1.26). 
Remark 1.33. We also have the following description of the canonical subgroups in the special fiber. Let $L$ be a finite extension of $\mathbb{Q}_{p}$, and let $x=(A, \lambda, \iota, \eta)$ be a $\mu$-ordinary point of $X$ defined over $O_{L}$. Let $A_{s}$ denote the special fiber of $A$; then the Frobenius $F$ acts on $A_{s}\left[\pi^{\infty}\right]$, and we can form the subgroups

$$
C_{i}:=\pi^{2 f-i} A_{s}\left[F^{2 f}, \pi^{2 f-i+1}\right]
$$

for $1 \leq i \leq 2 f$. Then we have $0 \subset C_{1} \subset \cdots \subset C_{2 f} \subset A_{s}[\pi]$, and the special fiber of each of the canonical subgroups is equal to one of the $C_{i}$. More precisely, we have $H_{j} \times k(L)=C_{r(j)}$ for $1 \leq j \leq s$, with $k(L)$ the residue field of $L$ and $r(j)=\min \left\{l: a_{l}=A_{j}\right\}$. Note also that $C_{i}^{\perp}=C_{2 f+1-i}$.

We can then define the relevant degree functions on $X_{\mathrm{Iw}, \pi}$. For each integer $k$, define

$$
d_{k}=\sum_{j=1}^{2 f} \min \left(\alpha_{j}, A_{k}\right) .
$$

Let $X_{\mathrm{Iw}, \pi \text {,rig }}$ be the associated rigid space. We define the degree function Deg : $X_{\mathrm{Iw}, \pi, \text { rig }} \rightarrow \prod_{k=1}^{s}\left[0, d_{k}\right]$ on this space by

$$
\operatorname{Deg}\left(A, \lambda, \iota, \eta, H_{\bullet}\right):=\left(\operatorname{deg} H_{A_{k}}\right)_{1 \leq k \leq s} .
$$

We also define the $k$-th degree function by $\operatorname{Deg}_{k}\left(A, \lambda, \iota, \eta, H_{\bullet}\right):=\operatorname{deg} H_{A_{k}}$ for $1 \leq k \leq s$. We then have the following description of the $\mu$-ordinary locus:

Proposition 1.34. The space $X_{\text {rig }}^{\mu \text {-ord }} \subset X_{\text {rig }}$ is exactly the image of

$$
\operatorname{Deg}^{-1}\left(\left\{d_{1}\right\} \times \cdots \times\left\{d_{s}\right\}\right)
$$

under the map $X_{\mathrm{Iw}, \pi, \text { rig }} \rightarrow X_{\text {rig. }}$.

Proof. If $x$ is a $\mu$-ordinary point, it follows from the previous corollary that there exist subgroups $H_{k}$ of height $2 f A_{k}$ with deg $H_{k}=d_{k}$ for all $1 \leq k \leq s$. Conversely, suppose that $(A, \lambda, \iota, \eta)$ is a point of $X_{\text {rig }}$, with $A$ defined over the ring of integers of an extension $L$ of $\mathbb{Q}_{p}$, and that there exist subgroups $H_{k}$ of height $2 f A_{k}$ with $\operatorname{deg} H_{k}=d_{k}$ for all $1 \leq k \leq s$. This implies that we have $\operatorname{deg}_{\sigma} H_{k}=\min \left(\alpha_{\sigma}, A_{k}\right)$ for all $\sigma \in D_{\pi}$ and $1 \leq k \leq s$. We want to show that $A$ is $\mu$-ordinary; it suffices to show that $A[\pi]$ has a nice description. Define $H_{0}=0, H_{i}=H_{2 s+1-i}^{\perp}$ for $s+1 \leq i \leq 2 s+1$, and let $H_{j}^{\prime}$ be a complement of $H_{j-1}$ in $H_{j}$ for all $1 \leq j \leq 2 s+1$. This is possible if the field $L$ is big enough. We claim that

$$
H_{j} \simeq H_{j}^{\prime} \times H_{j-1} .
$$

Indeed we have a morphism $H_{j-1} \rightarrow H_{j} / H_{j}^{\prime}$ that is an isomorphism in generic fiber. The degree of the image of $H_{j-1}$ in $H_{j} / H_{j}^{\prime}$ thus increases, but since the degree of 
$H_{j-1}$ is maximal, it must be an equality. We deduce that $\operatorname{deg} H_{j}=\operatorname{deg} H_{j}^{\prime}+\operatorname{deg} H_{j-1}$ and that the morphism $H_{j}^{\prime} \times H_{j-1} \rightarrow H_{j}$ is an isomorphism.

We finally get

$$
A[\pi] \simeq H_{1}^{\prime} \times H_{2}^{\prime} \times \cdots \times H_{2 s+1}^{\prime} .
$$

But we can explicitly describe the groups $H_{j}^{\prime}$ using the same proof as Proposition 1.29. We then conclude that $A$ is $\mu$-ordinary by Proposition 1.12 .

\section{Modular forms and Hecke operators}

2A. Modular forms. Let's now define the modular forms for the Shimura variety $X$. Let $\pi$ be a place of $F_{0}$ above $p$, and suppose it is in case (L). Then we define the $O_{F} \otimes O_{K}$-module $\mathrm{St}_{\pi}$ by

$$
\mathrm{St}_{\pi}:=O_{K}^{a_{\sigma}} \oplus O_{K}^{b_{\sigma}}
$$

where $O_{F}$ acts on $O_{K}^{a_{\sigma}}$ by $\sigma^{+}$and on $O_{K}^{b_{\sigma}}$ by $\sigma^{-}$. If $\pi$ is in case (U), we define the $O_{F} \otimes O_{K}$-module $\mathrm{St}_{\pi}$ by

$$
\mathrm{St}_{\pi}:=O_{K}^{a_{\sigma}} \oplus O_{K}^{b_{\sigma}}
$$

where $O_{F}$ acts on $O_{K}^{a_{\sigma}}$ by $\sigma_{1}$ and on $O_{K}^{b_{\sigma}}$ by $\sigma_{2}$. Finally, we define the $O_{F} \otimes O_{K^{-}}$ module St by

$$
\mathrm{St}=\bigoplus_{\pi} \mathrm{St}_{\pi},
$$

where $\pi$ runs over the places of $F_{0}$ above $p$. If $R$ is an $O_{K}$-algebra and $(A, \lambda, \iota, \eta)$ is an $R$-point of $X$, then the $R \otimes O_{F}$-module $e^{*} \Omega_{A / R}^{1}$ is isomorphic to St $\otimes O_{K} R$. The sheaf $\omega_{A}:=e^{*} \Omega_{A / X}^{1}$ is then locally isomorphic to $\operatorname{St} \otimes O_{K} \mathcal{O}_{X}$ (it is a locally free sheaf on $\mathcal{O}_{X}$ ).

Define

$$
\mathcal{T}=\operatorname{Isom}_{O_{F} \otimes \mathcal{O}_{X}}\left(\operatorname{St} \otimes \mathcal{O}_{X}, \omega_{A}\right) .
$$

This is a torsor on $X$ under the group, defined over $O_{K}$,

$$
M=\prod_{\pi \in \mathcal{P}} \prod_{\sigma \in \Sigma_{\pi}} \mathrm{GL}_{a_{\sigma}} \times \mathrm{GL}_{b_{\sigma}},
$$

where $\mathcal{P}$ is the set of primes of $F_{0}$ above $\pi$. Let $B_{M}$ be the upper Borel subgroup of $M, U_{M}$ its unipotent radical, and $T_{M}$ its maximal torus. Let $X\left(T_{M}\right)$ be the character group of $T_{M}$ and $X\left(T_{M}\right)^{+}$the cone of dominant weights for $B_{M}$. If $\kappa \in X\left(T_{M}\right)^{+}$, we let $\kappa^{\prime}=-w_{0} \kappa \in X\left(T_{M}\right)^{+}$, where $w_{0}$ is the element of highest length in the Weyl group of $M$ relative to $T_{M}$.

Let $\phi: \mathcal{T} \rightarrow X$ be the projection morphism. 
Definition 2.1. Let $\kappa \in X\left(T_{M}\right)^{+}$. The sheaf of modular forms of weight $\kappa$ is $\omega^{\kappa}=\phi_{*} O_{\mathcal{T}}\left[\kappa^{\prime}\right]$, where $\phi_{*} O_{\mathcal{T}}\left[\kappa^{\prime}\right]$ is the subsheaf of $\phi_{*} O_{\mathcal{T}}$, on which $B_{M}=T_{M} U_{M}$ acts by $\kappa^{\prime}$ on $T_{M}$ and trivially on $U_{M}$.

A modular form of weight $\kappa$ on $X$ with coefficients in an $O_{L}$-algebra $R$ is thus a global section of $\omega^{\kappa}$, so an element of $H^{0}\left(X \times O_{K} R, \omega^{\kappa}\right)$. Using the projection $X_{\mathrm{Iw}} \rightarrow X$, we similarly define the sheaf $\omega^{\kappa}$ on $X_{\mathrm{Iw}}$, as well as the modular forms on $X_{\text {Iw }}$.

2B. Overconvergent modular forms. For simplicity, we will now assume that there is only one place $\pi$ of $F_{0}$ above $p$, that is to say that $p$ is inert in $F_{0}$. The case with several places does not add any difficulty, and will be treated in Section 4.

We can then define the space of overconvergent modular forms. These will be sections of the sheaf of modular forms defined over a strict neighborhood of the $\mu$-ordinary locus. Recall that we have defined in both cases a degree function

$$
\text { Deg }: X_{\text {Iw,rig }} \rightarrow \prod_{k=1}^{s}\left[0, d_{k}\right] .
$$

Since there is only one place above $p$ in $F_{0}$, we have $X_{\mathrm{Iw}}=X_{\mathrm{Iw}, \pi}$.

We define the $\mu$-ordinary-multiplicative locus as $\operatorname{Deg}^{-1}\left(\left\{d_{1}\right\} \times \cdots \times\left\{d_{s}\right\}\right)$. By Proposition 1.29 or Proposition 1.34, this locus lies in the $\mu$-ordinary locus.

Definition 2.2. The space of overconvergent modular forms of weight $\kappa$ is defined as

$$
M^{\dagger}:=\operatorname{colim}_{\mathcal{V}} H^{0}\left(\mathcal{V}, \omega^{\kappa}\right)
$$

where $\mathcal{V}$ runs over the strict neighborhoods of the $\mu$-ordinary-multiplicative locus in $X_{\mathrm{Iw}, \text { rig. }}$.

An overconvergent modular form is then defined over a space of the form

$$
\operatorname{Deg}^{-1}\left(\left[d_{1}-\varepsilon, d_{1}\right] \times \cdots \times\left[d_{s}-\varepsilon, d_{s}\right]\right)
$$

for some $\varepsilon>0$.

2C. Hecke operators. We now define the Hecke operators. These operators will act both on the rigid space and on the space of modular forms. We will fix the weight $\kappa$. Explicitly, $\kappa$ is a collection of integers

$$
\left(\left(\kappa_{\sigma, 1} \geq \cdots \geq \kappa_{\sigma, a_{\sigma}}\right),\left(\lambda_{\sigma, 1} \geq \cdots \geq \lambda_{\sigma, b_{\sigma}}\right)\right)_{\sigma \in \Sigma_{\pi}} .
$$

We recall that we still assume that $\pi$ is the only place of $F_{0}$ above $p$. To simplify the notation, we define $\kappa_{\sigma}:=\kappa_{\sigma, a_{\sigma}}$ and $\lambda_{\sigma}:=\lambda_{\sigma, b_{\sigma}}$. 
2C1. Linear case. Assume that $\pi$ is in case (L). Let $1 \leq i \leq a+b-1$ be an integer, and let $C_{i}$ be the moduli space defined over $K$ parameterizing $\left(A, \lambda, \iota, \eta, H_{\bullet}, L\right)$, with $\left(A, \lambda, \iota, \eta, H_{\bullet}\right)$ a point of $X_{\mathrm{Iw}}$ and $L=L_{0} \oplus L_{0}^{\perp}$ a subgroup of $A[\pi]$, where $L_{0}$ is an $O_{F}$-stable subgroup of $A\left[\pi^{+}\right]$with $A\left[\pi^{+}\right]=H_{i} \oplus L_{0}$. We have two morphisms $p_{1}, p_{2}: C_{i} \rightarrow X_{\mathrm{Iw}} \times O_{K} K$. The morphism $p_{1}$ corresponds to forgetting $L$, and the morphism $p_{2}$ is defined as $p_{2}\left(A, \lambda, \iota, \eta, H_{\bullet}, L\right)=\left(A / L, \lambda^{\prime}, \iota^{\prime}, \eta^{\prime}, H_{\bullet}^{\prime}\right)$, with

- $H_{j}^{\prime}=\left(H_{j}+L_{0}\right) / L_{0}$ if $j \leq i$;

- $H_{j}^{\prime}=\left(\left(\pi^{+}\right)^{-1}\left(H_{j} \cap L_{0}\right)\right) / L_{0}$ if $j>i$.

We take the polarization $\lambda^{\prime}$ to be equal to $p \cdot \lambda$, which is a prime-to- $p$ polarization. Let $C_{i}^{\text {an }}$ be the analytic space associated to $C_{i}$, and define $C_{i, \text { rig }}:=p_{1}^{-1}\left(X_{\mathrm{Iw}, \mathrm{rig}}\right)$. The morphisms $p_{1}$ and $p_{2}$ give morphisms $C_{i, \text { rig }} \rightarrow X_{\text {Iw,rig. }}$

Definition 2.3. The $i$-th Hecke operator acting on the subsets of $X_{\mathrm{Iw}, \text { rig }}$ is defined by

$$
U_{\pi, i}(S)=p_{2}\left(p_{1}^{-1}(S)\right) .
$$

This operator preserves the admissible open subsets and quasicompact admissible open subsets.

Let us denote by $p: A \rightarrow A / L$ the universal isogeny over $C_{i}$. This induces an isomorphism $p^{*}: \omega_{(A / L) / X} \rightarrow \omega_{A / X}$, and thus a morphism $p^{*}(\kappa): p_{2}^{*} \omega^{\kappa} \rightarrow p_{1}^{*} \omega^{\kappa}$. For every admissible open $\mathcal{U}$ of $X_{\mathrm{Iw}, \text { rig }}$, we form the composed morphism

$$
\begin{aligned}
\widetilde{U}_{\pi, i}: H^{0}\left(U_{\pi, i}(\mathcal{U}), \omega^{\kappa}\right) \rightarrow H^{0}\left(p_{1}^{-1}(\mathcal{U}), p_{2}^{*} \omega^{\kappa}\right) & \\
& \stackrel{p^{*}(\kappa)}{\longrightarrow} H^{0}\left(p_{1}^{-1}(\mathcal{U}), p_{1}^{*} \omega^{\kappa}\right) \stackrel{\operatorname{Tr}_{p_{1}}}{\longrightarrow} H^{0}\left(\mathcal{U}, \omega^{\kappa}\right) .
\end{aligned}
$$

Definition 2.4. The Hecke operator acting on modular forms is defined by $U_{\pi, i}=$ $\left(1 / p^{N_{i}}\right) \widetilde{U}_{\pi, i}$ with

$$
N_{i}=\sum_{\sigma \in \Sigma_{\pi}}\left(\min \left(i, a_{\sigma}\right) \min \left(a+b-i, b_{\sigma}\right)+\max \left(a_{\sigma}-i, 0\right) \kappa_{\sigma}+\max \left(i-a_{\sigma}, 0\right) \lambda_{\sigma}\right) .
$$

We will also write

$$
n_{i}=\sum_{\sigma \in \Sigma_{\pi}} \min \left(i, a_{\sigma}\right) \min \left(a+b-i, b_{\sigma}\right)
$$

for the constant term of $N_{i}$, which is independent of the weight.

Let us explain briefly the meaning of the normalization factor $N_{i}$. The term $\min \left(i, a_{\sigma}\right) \min \left(a+b-i, b_{\sigma}\right)$ comes from the inseparability degree of the projection $p_{1}$. The term $\max \left(a_{\sigma}-i, 0\right) \kappa_{\sigma}+\max \left(i-a_{\sigma}, 0\right) \lambda_{\sigma}$ comes from the morphism $p^{*}(\kappa)$. Indeed, we have the following proposition: 
Proposition 2.5. Let $M$ be a finite extension of $\mathbb{Q}_{p}$, let $\left(A, \lambda, \iota, \eta, H_{\bullet}\right)$ be an $O_{M}$ point of $X_{\mathrm{Iw}}$ and let $L=L_{0} \oplus L_{0}^{\perp}$ be a subgroup of $A[\pi]$, where $L_{0}$ is an $O_{F}$-stable subgroup of $A\left[\pi^{+}\right]$with $A\left[\pi^{+}\right]=H_{i} \oplus L_{0}$ in generic fiber. Then we have, for all $\sigma \in \Sigma_{\pi}$,

$$
\operatorname{deg}_{\sigma} L_{0} \geq a_{\sigma}-i \quad \text { and } \quad \operatorname{deg}_{\sigma} L_{0}^{\perp} \geq i-a_{\sigma} .
$$

Proof. The group $A\left[\pi^{+}\right] / L_{0}$ is of height $f i$ and has partial degree $\left(a_{\sigma}-\operatorname{deg}_{\sigma} L_{0}\right)_{\sigma}$. Hence, $a_{\sigma}-\operatorname{deg}_{\sigma} L_{0} \leq i$ and $\operatorname{deg}_{\sigma} L_{0} \geq a_{\sigma}-i$. We get the other equality by duality (note that $\left.b_{\sigma}-(a+b-i)=i-a_{\sigma}\right)$.

We have the following proposition concerning the behavior of the Hecke operator regarding the degree function:

Proposition 2.6. Let $x=\left(A, \lambda, \iota, \eta, H_{0}\right)$ be a point of $X_{\mathrm{Iw}, \mathrm{rig}}$, and $y \in U_{\pi, i}(x)$ corresponding to a subgroup $L \in A[\pi]$ as before. Write $y=\left(A / L, \lambda, \iota, \eta, H_{\bullet}^{\prime}\right)$; then we have

$$
\operatorname{deg} H_{j}^{\prime} \geq \operatorname{deg} H_{j}
$$

for all $1 \leq j \leq a+b-1$. Moreover, we have

$$
\operatorname{deg} H_{i}^{\prime}=\operatorname{deg} A\left[\pi^{+}\right]-\operatorname{deg} L_{0} .
$$

If $\operatorname{deg} H_{i}^{\prime}=\operatorname{deg} H_{i}$, then $\operatorname{deg} H_{i} \in \mathbb{Z}$.

Proof. For all $1 \leq j \leq i$, the morphism $H_{j} \rightarrow H_{j}^{\prime}$ is an isomorphism in the generic fiber. Thus, $\operatorname{deg} H_{j}^{\prime} \geq \operatorname{deg} H_{j}$. If $i<j \leq a+b-1$, we have

$$
\operatorname{deg} H_{j}^{\prime}=\operatorname{deg}\left(\left(\pi^{+}\right)^{-1}\left(H_{j} \cap L_{0}\right)\right)-\operatorname{deg} L_{0} .
$$

Since $\operatorname{deg}\left(\left(\pi^{+}\right)^{-1} H\right)=\operatorname{deg} A\left[\pi^{+}\right]+\operatorname{deg} H$ for every subgroup $H$ of $A\left[\pi^{+}\right]$, we get

$$
\begin{aligned}
\operatorname{deg} H_{j}^{\prime} & =\operatorname{deg} A\left[\pi^{+}\right]+\operatorname{deg}\left(H_{j} \cap L_{0}\right)-\operatorname{deg} L_{0} \\
& =\operatorname{deg}\left(H_{j}+L_{0}\right)+\operatorname{deg}\left(H_{j} \cap L_{0}\right)-\operatorname{deg} L_{0} \geq \operatorname{deg} H_{j}
\end{aligned}
$$

from the properties of the degree function.

We have $H_{i}^{\prime}=\left(A\left[\pi^{+}\right] / L_{0}\right)$, hence the formula for the degree of $H_{i}^{\prime}$. If deg $H_{i}^{\prime}=$ $\operatorname{deg} H_{i}$, then we have $\operatorname{deg} H_{i}+\operatorname{deg} L_{0}=\operatorname{deg} A\left[\pi^{+}\right]$, and $A\left[\pi^{+}\right]=H_{i} \times L_{0}$. Since $A\left[\pi^{+}\right]$is a $\mathrm{BT}_{1}$, so is $H_{i}$, and its degree is an integer.

2C2. Unitary case. Assume now that $\pi$ is in case (U). Let $1 \leq i \leq \frac{1}{2}(a+b)$ be an integer, and let $C_{i}$ be the moduli space defined over $K$ parameterizing $\left(A, \lambda, \iota, \eta, H_{\bullet}, L\right)$, with $\left(A, \lambda, \iota, \eta, H_{\bullet}\right)$ a point of $X_{\mathrm{Iw}}$, where

- $L$ is an $O_{F}$-stable, totally isotropic subgroup of $A\left[\pi^{2}\right]$ such that $A[\pi]=$ $H_{i} \oplus L[\pi]=H_{i}^{\perp} \oplus \pi L$ if $i<\frac{1}{2}(a+b)$; 
- $L$ is an $O_{F}$-stable, totally isotropic subgroup of $A[\pi]$ such that $A[\pi]=H_{i} \oplus L$ if $i=\frac{1}{2}(a+b)$.

We have two morphisms $p_{1}, p_{2}: C_{i} \rightarrow X_{\mathrm{Iw}} \times O_{K} K$. The morphism $p_{1}$ corresponds to forgetting $L$, and the morphism $p_{2}$ is defined as $p_{2}\left(A, \lambda, \iota, \eta, H_{\bullet}, L\right)=$ $\left(A / L, \lambda^{\prime}, \iota^{\prime}, \eta^{\prime}, H_{\bullet}^{\prime}\right)$, with

- $H_{j}^{\prime}=\left(H_{j}+L\right) / L$ if $j \leq i$;

- $H_{j}^{\prime}=\left(\pi^{-1}\left(H_{j} \cap L\right)+L\right) / L$ if $i<j \leq \frac{1}{2}(a+b)$.

We take the polarization $\lambda^{\prime}$ to be equal to $p \cdot \lambda$, which is a prime-to- $p$ polarization. Let $C_{i}^{\text {an }}$ be the analytic space associated to $C_{i}$, and define $C_{i, \text { rig }}:=p_{1}^{-1}\left(X_{\mathrm{Iw}, \mathrm{rig}}\right)$. The morphisms $p_{1}$ and $p_{2}$ give morphisms $C_{i, \text { rig }} \rightarrow X_{\text {Iw,rig. }}$.

Definition 2.7. The $i$-th Hecke operator acting on the subsets of $X_{\text {Iw,rig }}$ is defined by

$$
U_{\pi, i}(S)=p_{2}\left(p_{1}^{-1}(S)\right) .
$$

This operator preserves the admissible open subsets and quasicompact admissible open subsets.

Remark 2.8. The condition $A[\pi]=H_{i}^{\perp} \oplus \pi L$ is actually redundant with the condition $A[\pi]=H_{i} \oplus L[\pi]$. Indeed, since $L$ is totally isotropic, we have $\pi L \subset L[\pi]^{\perp}$ (we denote by $L[\pi]^{\perp}$ the orthogonal in $A[\pi]$ of $L[\pi]$ ). Comparing the heights, we see that we have the equality $\pi L=L[\pi]^{\perp}$.

Let us denote by $p: A \rightarrow A / L$ the universal isogeny over $C_{i}$. This induces an isomorphism $p^{*}: \omega_{(A / L) / X} \rightarrow \omega_{A / X}$, and thus a morphism $p^{*}(\kappa): p_{2}^{*} \omega^{\kappa} \rightarrow p_{1}^{*} \omega^{\kappa}$. For every admissible open subset $\mathcal{U}$ of $X_{\mathrm{Iw}, \text { rig }}$, we form the composed morphism

$\widetilde{U}_{\pi, i}: H^{0}\left(U_{\pi, i}(\mathcal{U}), \omega^{\kappa}\right) \rightarrow H^{0}\left(p_{1}^{-1}(\mathcal{U}), p_{2}^{*} \omega^{\kappa}\right)$

$$
\stackrel{p^{*}(\kappa)}{\longrightarrow} H^{0}\left(p_{1}^{-1}(\mathcal{U}), p_{1}^{*} \omega^{\kappa}\right) \stackrel{\operatorname{Tr}_{p_{1}}}{\longrightarrow} H^{0}\left(\mathcal{U}, \omega^{\kappa}\right) .
$$

Definition 2.9. The Hecke operator acting on modular forms is defined by $U_{\pi, i}=$ $\left(1 / p^{N_{i}}\right) \widetilde{U}_{\pi, i}$ with

$$
N_{i}=\sum_{\sigma \in \Sigma_{\pi}}\left((a+b) \min \left(i, a_{\sigma}\right)+\max \left(a_{\sigma}-i, 0\right) \kappa_{\sigma}+\max \left(b_{\sigma}-a_{\sigma}, b_{\sigma}-i\right) \lambda_{\sigma}\right)
$$

if $i<\frac{1}{2}(a+b)$, and

$$
N_{(a+b) / 2}=\sum_{\sigma \in \Sigma_{\pi}}\left(\frac{1}{2}(a+b) a_{\sigma}+\frac{1}{2}\left(b_{\sigma}-a_{\sigma}\right) \lambda_{\sigma}\right) .
$$

We will also write

$$
n_{i}=\sum_{\sigma \in \Sigma_{\pi}}(a+b) \min \left(i, a_{\sigma}\right)
$$


if $i<\frac{1}{2}(a+b)$, and

$$
n_{(a+b) / 2}=\sum_{\sigma \in \Sigma_{\pi}} \frac{1}{2}(a+b) a_{\sigma}
$$

for the constant term of $N_{i}$, which is independent of the weight.

Again, the reason for the normalization factor $n_{i}$ comes in two parts. The term $(a+b) \min \left(i, a_{\sigma}\right)$ comes from the inseparability degree of the projection $p_{1}$. The term $\max \left(a_{\sigma}-i, 0\right) \kappa_{\sigma}+\max \left(b_{\sigma}-a_{\sigma}, b_{\sigma}-i\right) \lambda_{\sigma}$ comes from the morphism $p^{*}(\kappa)$. Indeed, we have the following proposition:

Proposition 2.10. Let $M$ be a finite extension of $\mathbb{Q}_{p}$, let $\left(A, \lambda, \iota, \eta, H_{\bullet}\right)$ be an $O_{M}$-point of $X_{\mathrm{Iw}}$ and let $L$ be a subgroup of $A\left[\pi^{2}\right]$ as before. If $i<\frac{1}{2}(a+b)$, we have

$$
\operatorname{deg}_{\sigma_{1}} L \geq a_{\sigma}-i \quad \text { and } \quad \operatorname{deg}_{\sigma_{2}} L \geq \max \left(b_{\sigma}-i, b_{\sigma}-a_{\sigma}\right) .
$$

If $i=\frac{1}{2}(a+b)$, we have

$$
\operatorname{deg}_{\sigma_{2}} L \geq \frac{1}{2}\left(b_{\sigma}-a_{\sigma}\right) .
$$

Proof. Suppose first that $i<\frac{1}{2}(a+b)$. The subgroup $L$ being totally isotropic, we get

$$
\operatorname{deg}_{\sigma_{2}} L=\operatorname{deg}_{\sigma_{1}} L+b_{\sigma}-a_{\sigma}
$$

The group $A[\pi] / L[\pi]$ is of height $2 f i$, hence we have $\operatorname{deg}_{\sigma_{1}} A[\pi] / L[\pi] \leq i$. We get

$$
\operatorname{deg}_{\sigma_{1}} L \geq \operatorname{deg}_{\sigma_{1}} L[\pi] \geq a_{\sigma}-i \text {. }
$$

We deduce that $\operatorname{deg}_{\sigma_{2}} L=\operatorname{deg}_{\sigma_{1}} L+b_{\sigma}-a_{\sigma} \geq \max \left(b_{\sigma}-i, b_{\sigma}-a_{\sigma}\right)$.

If $i=\frac{1}{2}(a+b)$, then $L$ is a maximal totally isotropic subgroup of $A[\pi]$. Thus, we have

$$
\operatorname{deg}_{\sigma_{2}} L=\frac{1}{2}\left(b_{\sigma}-a_{\sigma}\right)+\operatorname{deg}_{\sigma_{1}} L \geq \frac{1}{2}\left(b_{\sigma}-a_{\sigma}\right) .
$$

We have the following proposition concerning the behavior of the Hecke operator regarding the degree function:

Proposition 2.11. Let $x=\left(A, \lambda, \iota, \eta, H_{\bullet}\right)$ be a point of $X_{\mathrm{Iw}, \mathrm{rig}}$, and $y \in U_{\pi, i}(x)$ corresponding to a subgroup $L \in A\left[\pi^{2}\right]$ as before. Write $y=\left(A / L, \lambda, \iota, \eta, H_{\bullet}^{\prime}\right)$; then we have

$$
\operatorname{deg} H_{j}^{\prime} \geq \operatorname{deg} H_{j}
$$

for all $1 \leq j \leq \frac{1}{2}(a+b)$. Moreover, if $i<\frac{1}{2}(a+b)$, we have

$$
\operatorname{deg} H_{i}^{\prime}=2 f i-\operatorname{deg}(L / L[\pi]) .
$$

If $\operatorname{deg} H_{i}^{\prime}=\operatorname{deg} H_{i}$, then $\operatorname{deg} H_{i} \in \mathbb{Z}$. 
If $i=\frac{1}{2}(a+b)$, then

$$
\operatorname{deg} H_{(a+b) / 2}^{\prime}=f(a+b)-\operatorname{deg}(L) .
$$

If $\operatorname{deg} H_{(a+b) / 2}^{\prime}=\operatorname{deg} H_{(a+b) / 2}$, then $d_{(a+b) / 2}-\operatorname{deg} H_{(a+b) / 2} \in 2 \mathbb{Z}$.

Proof. Suppose first that $i<\frac{1}{2}(a+b)$. For each $1 \leq j \leq i$, the morphism $H_{j} \rightarrow H_{j}^{\prime}$ is an isomorphism in the generic fiber. Thus, $\operatorname{deg} H_{j}^{\prime} \geq \operatorname{deg} H_{j}$. Suppose $i<j \leq$ $\frac{1}{2}(a+b)$. Then, observing that $\pi^{-1}\left(H_{j} \cap L\right) \cap L=L[\pi]$, we get

$$
\begin{aligned}
\operatorname{deg} H_{j}^{\prime} & =\operatorname{deg}\left(\pi^{-1}\left(H_{j} \cap L\right)+L\right)-\operatorname{deg} L \\
& \geq \operatorname{deg}\left(\pi^{-1}\left(H_{j} \cap L\right)\right)-\operatorname{deg} L[\pi] \\
& \geq \operatorname{deg} A[\pi]+\operatorname{deg}\left(H_{j} \cap L\right)-\operatorname{deg} L[\pi] \\
& =\operatorname{deg}\left(H_{j}+L[\pi]\right)+\operatorname{deg}\left(H_{j} \cap L[\pi]\right)-\operatorname{deg} L[\pi] \\
& \geq \operatorname{deg} H_{j}
\end{aligned}
$$

from the properties of the degree function.

Let us calculate the degree of $H_{i}^{\prime}$. We have

$$
\begin{aligned}
\operatorname{deg} H_{i}^{\prime} & =\operatorname{deg}(A[\pi]+L) / L \\
& =\operatorname{deg}\left(\pi^{-1} L[\pi]^{\perp}\right) / L=\operatorname{deg} A[\pi]+\operatorname{deg} L[\pi]^{\perp}-\operatorname{deg} L \\
& =2 f i+\operatorname{deg} L[\pi]-\operatorname{deg} L=2 f i-\operatorname{deg}(L / L[\pi]) .
\end{aligned}
$$

If $\operatorname{deg} H_{i}^{\prime}=\operatorname{deg} H_{i}$, then we have $\operatorname{deg} H_{i}+\operatorname{deg} L[\pi]=\operatorname{deg} A[\pi]$ and $A[\pi]=$ $H_{i} \times L[\pi]$. Since $A[\pi]$ is a $\mathrm{BT}_{1}$, so is $H_{i}$, and its degree is an integer.

Suppose now that $i=\frac{1}{2}(a+b)$. The same argument as before shows that we still have $\operatorname{deg} H_{j}^{\prime} \geq \operatorname{deg} H_{j}$ for all $1 \leq j \leq \frac{1}{2}(a+b)$. Since $H_{(a+b) / 2}^{\prime}=A[\pi] / L$, we have

$$
\operatorname{deg} H_{(a+b) / 2}^{\prime}=\operatorname{deg} A[\pi]-\operatorname{deg} L=f(a+b)-\operatorname{deg} L .
$$

If we have the equality $\operatorname{deg} H_{(a+b) / 2}^{\prime}=\operatorname{deg} H_{(a+b) / 2}$, then $H_{(a+b) / 2}$ is a $\mathrm{BT}_{1}$, and all its partial degrees are integers. Since $H_{(a+b) / 2}$ is totally isotropic, we have the relations

$$
\operatorname{deg}_{\sigma_{2}} H_{(a+b) / 2}=\operatorname{deg}_{\sigma_{1}} H_{(a+b) / 2}+\frac{1}{2}\left(b_{\sigma}-a_{\sigma}\right)
$$

for all $\sigma \in \Sigma_{\pi}$. If we write $h_{\sigma}=\operatorname{deg}_{\sigma_{1}} H_{(a+b) / 2}$, which is an integer under the previous assumption, we have

$$
\begin{aligned}
d_{(a+b) / 2}-\operatorname{deg} H_{(a+b) / 2} & =\sum_{\sigma \in \Sigma_{\pi}}\left(a_{\sigma}+\frac{1}{2}(a+b)-2 h_{\sigma}-\frac{1}{2}\left(b_{\sigma}-a_{\sigma}\right)\right) \\
& =2 \sum_{\sigma \in \Sigma_{\pi}}\left(a_{\sigma}-h_{\sigma}\right) \in 2 \mathbb{Z} .
\end{aligned}
$$

We will also need the following useful lemma: 
Lemma 2.12. Let $x=\left(A, \lambda, \iota, \eta, H_{\bullet}\right)$ be a point of $X_{\mathrm{Iw}, \mathrm{rig}}$, and let $L \in A\left[\pi^{2}\right]$ be a totally isotropic subgroup as before, corresponding to a point of $U_{\pi, i}(x)$, with $i<\frac{1}{2}(a+b)$. We have the inequality

$$
\operatorname{deg}(L / L[\pi]) \leq 2 f i-\operatorname{deg} A[\pi]+\operatorname{deg} L[\pi] .
$$

Proof. The multiplication by $\pi$ gives a morphism $L / L[\pi] \rightarrow \pi L$. But, $L$ being totally isotropic, we have the relation $\pi L=L[\pi]^{\perp}$. We thus get a morphism $L / L[\pi] \rightarrow L[\pi]^{\perp}$, which is an isomorphism in generic fiber. Thus,

$$
\operatorname{deg}(L / L[\pi]) \leq \operatorname{deg} L[\pi]^{\perp}=2 f i-\operatorname{deg} A[\pi]+\operatorname{deg} L[\pi] .
$$

\section{A classicality result}

We will now prove a control theorem, that is to say that an overconvergent modular form is indeed classical under a certain assumption.

3A. Decomposition of the Hecke operators. Fix a rational $\varepsilon>0$. We will fix rationals $\varepsilon_{k} \in\left\{\varepsilon, d_{k}\right\}$ for $1 \leq k \leq s$. Also fix an integer $i$ between 1 and $s$ such that $\varepsilon_{i}=\varepsilon$. Since we have assumed that there is only one place $\pi$ of $F_{0}$ above $p$, we will simply let $\Sigma=\Sigma_{\pi}$. We define a partition of this set. First, we let, for $1 \leq k \leq s$,

$$
\Sigma_{k}:=\left\{\sigma \in \Sigma: a_{\sigma}=A_{k}\right\} .
$$

We will also let $\Sigma_{0}:=\left\{\sigma \in \Sigma: a_{\sigma}=0\right\}$ and $\Sigma_{s+1}:=\left\{\sigma \in \Sigma: a_{\sigma}=a+b\right\}$ (of course $\Sigma_{s+1}$ is always empty in case (U)). The sets $\left(\Sigma_{k}\right)_{0 \leq k \leq s+1}$ form a partition of $\Sigma$.

From the collection $\left(\varepsilon_{k}\right)_{1 \leq k \leq s}$, we define another partition of $\Sigma$. The set $S_{1}$ is defined to be

$$
S_{1}=\Sigma_{0} \cup \bigcup_{\substack{k \neq i \\ \varepsilon_{k}=\varepsilon}} \Sigma_{k} \cup \Sigma_{s+1} .
$$

The complement is the set

$$
S_{2}=\Sigma_{i} \cup \bigcup_{k, \varepsilon_{k}=d_{k}} \Sigma_{k}
$$

Define

$$
\begin{aligned}
\mathcal{U}_{0} & :=\operatorname{Deg}^{-1}\left(\left[d_{1}-\varepsilon_{1}, d_{1}\right] \times \cdots \times\left[d_{s}-\varepsilon_{s}, d_{s}\right]\right)=\bigcap_{k=1}^{s} \operatorname{Deg}_{k}^{-1}\left[d_{k}-\varepsilon_{k}, d_{k}\right], \\
\mathcal{U}_{1} & :=\bigcap_{k \neq i} \operatorname{Deg}_{k}^{-1}\left[d_{k}-\varepsilon_{k}, d_{k}\right] .
\end{aligned}
$$

We will define a decomposition of the Hecke operator $U_{\pi, A_{i}}$ on subsets of $\mathcal{U}_{1}$. Fix a rational $\alpha>0$, and define the integer $t$ to be 1, except in the unitary case 
and if $i=\frac{1}{2}(a+b)$, where we set $t=2$. For simplicity, we will write $U_{i}$ for $U_{\pi, A_{i}}$. Define

$$
\mathcal{U}:=\operatorname{Deg}_{i}^{-1}\left(\left[0, d_{i}-t(1-\alpha)\right]\right) \cap \mathcal{U}_{1} .
$$

Theorem 3.1. Let $N \geq 1$ and let $\beta<\varepsilon$ be a positive rational. There exists a finite ordered set $M_{N}$ and a decreasing sequence of admissible open subsets $\left(\mathcal{U}_{k}(N)\right)_{k \in M_{N}}$ of $\mathcal{U}$ such that, for all $k \geq 0$, we have a decomposition of the operator $U_{i}^{N}$ on $\mathcal{U}_{k}(N) \backslash \mathcal{U}_{k+1}(N)$ of the form

$$
U_{i}^{N}=\left(\coprod_{j=0}^{N-1} U_{i}^{N-1-j} \circ T_{j}\right) \amalg T_{N},
$$

with $T_{0}=U_{i, k, N}^{\text {good }}$,

$$
T_{j}=\coprod_{k_{1} \in M_{N-1}, \ldots, k_{j} \in M_{N-j}} U_{i, k_{j}, N}^{\mathrm{good}} U_{i, k_{j-1}, k_{j}, N}^{\mathrm{bad}} \ldots U_{i, k, k_{1}, N}^{\mathrm{bad}} \quad \text { for } 0<j<N,
$$

and

$$
T_{N}=\coprod_{k_{1} \in M_{N-1}, \ldots, k_{N-1} \in M_{1}} U_{i, k_{N-1}, N}^{\mathrm{bad}} U_{i, k_{N-2}, k_{N-1}, N}^{\mathrm{bad}} \ldots U_{i, k, k_{1}, N}^{\mathrm{bad}}
$$

such that

- the images of $U_{i, j, N}^{\mathrm{good}}$ for $j \in M_{k}$ are in $\left.\left.\operatorname{Deg}_{i}^{-1}(] d_{i}-t(1-\beta), d_{i}\right]\right)$;

- the images of $U_{i, l, l^{\prime}, N}^{\mathrm{bad}}$ for $l \in M_{k}$ and $l^{\prime} \in M_{k-1}$, and $U_{i, l, N}^{\mathrm{bad}}$ for $l \in M_{1}$ are in $\operatorname{Deg}_{i}^{-1}\left(\left[0, d_{i}-t(1-\beta)\right]\right)$.

The idea is that the points in $\left.] d_{i}-t(1-\beta), d_{i}\right]$ are "good" (because the overconvergent modular form will be defined at these points), and the points in $\operatorname{Deg}_{i}^{-1}\left(\left[0, d_{i}-t(1-\beta)\right]\right)$ are "bad". The decomposition in the theorem is then made to ensure that all the operators have their image either in good points or in bad points.

Let us describe this decomposition by looking at a point $x \in \mathcal{U}$. The set $U_{i}(\{x\})$ is finite and has, say, $N_{1}$ points in $\operatorname{Deg}_{i}^{-1}\left(\left[0, d_{i}-t(1-\beta)\right]\right)$ and $N_{2}$ in its complement $\left.\left.\operatorname{Deg}_{i}^{-1}(] d_{i}-t(1-\beta), d_{i}\right]\right)$. Thus, one can decompose the operator $U_{i}$ over $x$ as

$$
U_{i}=U_{i, x}^{\mathrm{good}} \amalg U_{i, x}^{\mathrm{bad}}
$$

with $U_{i, x}^{\text {bad }}$ corresponding to the $N_{1}$ points in $\operatorname{Deg}_{i}^{-1}\left(\left[0, d_{i}-t(1-\beta)\right]\right)$ and $U_{i, x}^{\text {good }}$ to the $N_{2}$ other points. This is a decomposition of operators, meaning that the set $U_{i}(\{x\})$ is the disjoint union of the sets $U_{i, x}^{\text {good }}(\{x\})$ and $U_{i, x}^{\mathrm{bad}}(\{x\})$; moreover, the operators $U_{i, x}^{\text {good }}$ and $U_{i, x}^{\text {bad }}$ induce morphisms $H^{0}\left(U_{i}(\{x\}), \omega^{\kappa}\right) \rightarrow H^{0}\left(\{x\}, \omega^{\kappa}\right)$ for each weight $\kappa$, such that

$$
U_{i} f=U_{i, x}^{\mathrm{good}} f+U_{i, x}^{\mathrm{bad}} f
$$


for all $f \in H^{0}\left(U_{i}(\{x\}), \omega^{\kappa}\right)$. These morphisms are defined in the same way as the morphism $U_{i}$, with the same normalization factor. This gives the decomposition of the theorem for $N=1$ at the point $x$.

To get the decomposition for $N=2$, one needs to study the operator $U_{i, x}^{\mathrm{bad}}$. Let $x_{1}, \ldots, x_{N_{1}}$ be the elements of the set $U_{i, x}^{\mathrm{bad}}(\{x\})$. We can thus decompose the operator $U_{i, x}^{\text {bad }}$ into

$$
U_{i, x}^{\mathrm{bad}}=\coprod_{j=1}^{N_{1}} U_{i, x, j}^{\mathrm{bad}},
$$

where $U_{i, x, j}^{\mathrm{bad}}$ corresponds to the point $x_{j}$. Then, for each $1 \leq j \leq N_{1}$, we have the decomposition of the operator $U_{i}$ at the point $x_{j}$ as $U_{i, x_{j}}^{\mathrm{good}} U U_{i, x_{j}}^{\mathrm{bad}}$. One then gets the decomposition of $U_{i}^{2}$ at the point $x$,

$$
U_{i}^{2}=U_{i} \circ U_{i, x}^{\mathrm{good}} \amalg \coprod_{j=1}^{N_{1}} U_{i, x_{j}}^{\mathrm{good}} \circ U_{i, x, j}^{\mathrm{bad}} \amalg \coprod_{j=1}^{N_{1}} U_{i, x_{j}}^{\mathrm{bad}} \circ U_{i, x, j}^{\mathrm{bad}} .
$$

Repeating this argument, one gets the desired decomposition of the operator $U_{i}^{N}$ at the point $x$. Of course, this decomposition does not have a meaning on the whole open subset $\mathcal{U}$ because the morphisms used to define the different operators will not be finite (the integer $N_{1}$ depends on the point $x$ ). But, on an adequate subset, this will be the case. That is why one has to construct the subsets $\left(\mathcal{U}_{k}(N)\right)_{k \in M_{N}}$. This construction and the proof of its properties have been done in [Bijakowski et al. 2016, Theorem 4.4.1].

3B. Analytic continuation. Let $f$ be a section of the sheaf $\omega^{\kappa}$ on $\mathcal{U}_{0}$. We will show that $f$ can be extended to $\mathcal{U}_{1}$ under a certain condition. More precisely, suppose in this section that $f$ is an eigenform for the Hecke operator $U_{i}$ with eigenvalue $\alpha_{i}$ and that

$$
v\left(\alpha_{i}\right)+n_{A_{i}}<(1-2 f \varepsilon) \inf _{\sigma \in S_{2}}\left(\kappa_{\sigma}+\lambda_{\sigma}\right) .
$$

The first step is to extend $f$ to $\left.\left.\operatorname{Deg}_{i}^{-1}(] d_{i}-t, d_{i}\right]\right) \cap \mathcal{U}_{1}$. We will use the following proposition:

Proposition 3.2. Let $0<\gamma<1$ be a rational. Then there exists an integer $N$ such that

$$
U_{i}^{N}\left(\operatorname{Deg}_{i}^{-1}\left(\left[d_{i}-t \gamma, d_{i}\right]\right) \cap \mathcal{U}_{1}\right) \subset \operatorname{Deg}_{i}^{-1}\left(\left[d_{i}-\varepsilon, d_{i}\right]\right) \cap \mathcal{U}_{1} .
$$

Proof. The key point is that on $\operatorname{Deg}_{i}^{-1}\left(\left[d_{i}-t \gamma, d_{i}\right]\right) \cap \mathcal{U}_{1}$ the operator $U_{i}$ strictly increases the degree function $\operatorname{Deg}_{i}$. Since $\operatorname{Deg}_{i}^{-1}\left(\left[d_{i}-t \gamma, d_{i}\right]\right) \cap \mathcal{U}_{1}$ is a quasicompact open subset of $X_{\mathrm{Iw}, \text { rig }}$, one can then apply the argument in [Pilloni 2011, Proposition 2.5].

Corollary 3.3. The overconvergent form $f$ extends to $\left.\left.\operatorname{Deg}_{i}^{-1}(] d_{i}-t, d_{i}\right]\right) \cap \mathcal{U}_{1}$. 
Proof. Let $0<\gamma<1$ be a rational. By the previous proposition, there exists an integer $N$ such that

$$
U_{i}^{N}\left(\operatorname{Deg}_{i}^{-1}\left(\left[d_{i}-t \gamma, d_{i}\right]\right) \cap \mathcal{U}_{1}\right) \subset \operatorname{Deg}_{i}^{-1}\left(\left[d_{i}-\varepsilon, d_{i}\right]\right) \cap \mathcal{U}_{1} .
$$

The quantity $\alpha_{i}^{-N} U_{i}^{N} f$ is thus defined on $\operatorname{Deg}_{i}^{-1}\left(\left[d_{i}-t \gamma, d_{i}\right]\right) \cap \mathcal{U}_{1}$. This formula allows us to extend $f$ to $\left.\left.\operatorname{Deg}_{i}^{-1}(] d_{i}-t, d_{i}\right]\right) \cap \mathcal{U}_{1}$.

The second step is to define some series on $\mathcal{U}:=\operatorname{Deg}_{i}^{-1}\left(\left[0, d_{i}-t(1-\alpha)\right]\right) \cap \mathcal{U}_{1}$ for some sufficiently small rational $\alpha$. We will use the decomposition of the Hecke operator $U_{i}$. First, we recall the definition of the norm of an operator. If $\mathcal{U}$ is an

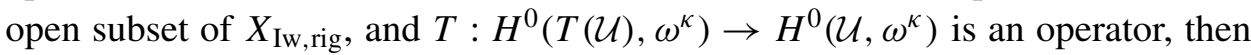
we define the norm of $T$ as

$$
\|T\|:=\inf \left\{r>0:|T f|_{\mathcal{U}} \leq r|f|_{T(\mathcal{U})} \text { for all } f \in H^{0}\left(T(\mathcal{U}), \omega^{\kappa}\right)\right\} .
$$

Theorem 3.4. Suppose that all the operators $U_{i}^{\mathrm{bad}}$ introduced in Theorem 3.1 satisfy the relation

$$
\left\|\alpha_{i}^{-1} U_{i}^{\mathrm{bad}}\right\|<1 .
$$

Then it is possible to construct sections $f_{N} \in H^{0}\left(\mathcal{U}, \omega^{\kappa} / p^{A_{N}}\right)$ such that $A_{N} \rightarrow \infty$. Moreover, the functions $f_{N}$ can be glued together with the initial form $f$ to give an element of $H^{0}\left(\mathcal{U}_{1}, \omega^{\kappa}\right)$.

The construction of the series $f_{N}$ and the proof of the gluing process have been done in [Bijakowski et al. 2016, Section 4.5]. Let us briefly describe the method. We take an element $\beta>0$ and consider the open subsets $\left(\mathcal{U}_{k}(N)\right)_{k \in M_{N}}$ constructed in Theorem 3.1 for this element. Let $K_{N}$ be the largest element of $M_{N}$; neglecting the bad points for the operator $U_{i}^{N}$ gives a function $g_{K_{N}} \in H^{0}\left(\mathcal{U}_{K_{N}}(N), \omega^{K}\right)$ (we refer to [Bijakowski et al. 2016, Definition 4.5.2] for the precise definition of the function $g_{K_{N}}$. Now take $\beta^{\prime}<\beta$, and again apply Theorem 3.1 for this other element: we get open subsets $\left(\mathcal{U}_{k}(N)^{\prime}\right)_{k \in M_{N}}$. We consider the subset $\mathcal{U}_{K_{N}-1}(N)^{\prime} \backslash \mathcal{U}_{K_{N}}(N)^{\prime}$ and the decomposition of $U_{i}^{N}$ on it. Neglecting the bad points gives a function $g_{K_{N}-1} \in H^{0}\left(\mathcal{U}_{K_{N}-1}(N)^{\prime} \backslash \mathcal{U}_{K_{N}}(N)^{\prime}, \omega^{\kappa}\right)$. One can then show that $g_{K_{N}}$ and $g_{K_{N}-1}$ can be glued together to give a function modulo $p^{A_{N}}$, where $A_{N}$ is an explicit constant. One thus gets a function $f_{N} \in H^{0}\left(\mathcal{U}_{K_{N}-1}(N)^{\prime}, \omega^{\kappa} / p^{A_{N}}\right)$ (see [Bijakowski et al. 2016, Proposition 4.5.6] for more details). Repeating this argument, one thus extends the function $f_{N}$ to the whole $\mathcal{U}$. The hypothesis on the operators implies that the sequence $\left(A_{N}\right)_{N}$ tends to infinity. A gluing lemma ([Bijakowski et al. 2016, Proposition 4.5.7], following [Kassaei 2006]) then ensures the existence of a function $f \in H^{0}\left(\mathcal{U}, \omega^{\kappa}\right)$, which can be glued with the initial function $f$, thus getting a section on $\mathcal{U}_{1}$.

We will now prove that, under the assumption made at the beginning of Section 3B, the condition in the theorem is fulfilled, that is to say that the norm of the operators 
$\alpha_{i}^{-1} U_{i}^{\text {bad }}$ is strictly less than 1 . We will split the discussion between the linear and unitary cases.

3B1. Linear case. We recall that the integer $t$ is equal to 1 in this case. Let $M$ be a finite extension of $\mathbb{Q}_{p}$, let $x=\left(A, \lambda, \iota, \eta, H_{\bullet}\right)$ be a point of $\mathcal{U}$ defined over $O_{M}$, and let $L=L_{0} \oplus L_{0}^{\perp}$ be a subgroup of $A[\pi]$, where $L_{0}$ is an $O_{F}$-stable subgroup of $A\left[\pi^{+}\right]$with $A\left[\pi^{+}\right]=H_{A_{i}} \oplus L_{0}$ in generic fiber. Let us write $A^{\prime}=A / L$, and let $H_{A_{i}}^{\prime}$ be the image of $H_{A_{i}}$ in $A / L$. Thus,

$$
H_{A_{i}}^{\prime}=A\left[\pi^{+}\right] / L_{0} .
$$

We suppose that the subgroup $L$ corresponds to a bad point, that is to say that $\operatorname{deg} H_{A_{i}}^{\prime} \leq d_{i}-1+\alpha$ for a certain rational $\alpha>0$. We write

$$
\operatorname{deg}_{\sigma} L_{0}=\max \left(a_{\sigma}-A_{i}, 0\right)+l_{\sigma}
$$

for all $\sigma \in \Sigma$. As is shown by Proposition 2.5, $l_{\sigma}$ is a positive rational for all $\sigma$. We then have, for all $\sigma$,

$$
\operatorname{deg}_{\sigma} H_{A_{i}}^{\prime}=a_{\sigma}-\operatorname{deg}_{\sigma} L_{0}=\min \left(A_{i}, a_{\sigma}\right)-l_{\sigma} .
$$

We deduce that $\operatorname{deg} H_{A_{i}}^{\prime}=d_{i}-\sum_{\sigma \in \Sigma} l_{\sigma}$. The condition of being a bad point gives

$$
\sum_{\sigma \in \Sigma} l_{\sigma} \geq 1-\alpha
$$

Actually, we can control some of the $l_{\sigma}$. First, we prove the following technical lemma:

Lemma 3.5. Let $x=\left(A, \lambda, \iota, \eta, H_{\bullet}\right)$ be a point as before and let $H \subset A\left[\pi^{+}\right]$ be an $O_{F}$-stable subgroup. If $H$ is of height $f A_{k}$ and $\operatorname{deg} H \geq d_{k}-\varepsilon$, then $\operatorname{deg}_{\sigma} H \geq \min \left(a_{\sigma}, A_{k}\right)-\varepsilon$ for all $\sigma \in \Sigma$.

If $H$ is of height $f\left(a+b-A_{k}\right)$ and $\operatorname{deg} H \leq \operatorname{deg} A\left[\pi^{+}\right]-d_{k}+\varepsilon$, then $\operatorname{deg}_{\sigma} H \leq$ $\max \left(a_{\sigma}-A_{k}, 0\right)+\varepsilon$ for all $\sigma \in \Sigma$.

Proof. Suppose that $H$ is of height $f A_{k}$ and $\operatorname{deg} H \geq d_{k}-\varepsilon$. If $\operatorname{deg}_{\sigma} H<$ $\min \left(a_{\sigma}, A_{k}\right)-\varepsilon$ for some $\sigma \in \Sigma$, then

$$
\operatorname{deg} H=\sum_{\sigma^{\prime} \in \Sigma} \operatorname{deg}_{\sigma^{\prime}} H<\min \left(a_{\sigma}, A_{k}\right)-\varepsilon+\sum_{\sigma^{\prime} \neq \sigma} \min \left(a_{\sigma^{\prime}}, A_{k}\right)=d_{k}-\varepsilon
$$

and we get a contradiction.

If $H$ is of height $f\left(a+b-A_{k}\right)$ and $\operatorname{deg} H \leq \operatorname{deg} A\left[\pi^{+}\right]-d_{k}+\varepsilon$, then we can apply the previous argument to $A\left[\pi^{+}\right] / H$. We get $\operatorname{deg}_{\sigma}\left(A\left[\pi^{+}\right] / H\right) \geq \min \left(a_{\sigma}, A_{k}\right)-\varepsilon$ for all $\sigma \in \Sigma$, and therefore $\operatorname{deg}_{\sigma} H \leq \max \left(a_{\sigma}-A_{k}, 0\right)+\varepsilon$.

Now we prove a bound for some of the $l_{\sigma}$. 
Lemma 3.6. If $\sigma \in S_{1}$, we have $l_{\sigma} \leq \varepsilon$.

Proof. If $\sigma \in \Sigma_{0} \cup \Sigma_{s+1}$, then $l_{\sigma}=0$. If not, $\sigma \in \Sigma_{k}$, with $1 \leq k \leq s, k \neq i$, and $\varepsilon_{k}=\varepsilon$. Since $x$ is a point of $\mathcal{U}$, we have $\operatorname{deg} H_{A_{k}} \geq d_{k}-\varepsilon$. Suppose first that $k<i$. Since $H_{A_{k}}$ and $L_{0}$ are disjoint, the morphism $H_{A_{k}} \rightarrow H_{A_{k}}^{\prime}:=\left(H_{A_{k}}+L_{0}\right) / L_{0}$ is an isomorphism in the generic fiber. Thus, by the properties of the degree function, $\operatorname{deg} H_{A_{k}}^{\prime} \geq \operatorname{deg} H_{A_{k}} \geq d_{k}-\varepsilon$. By Lemma 3.5, we get $\operatorname{deg}_{\sigma} H_{A_{k}}^{\prime} \geq A_{k}-\varepsilon$. But we also have $\operatorname{deg}_{\sigma} H_{A_{k}}^{\prime} \leq \operatorname{deg}_{\sigma} H_{A_{i}}^{\prime}=\min \left(A_{i}, a_{\sigma}\right)-l_{\sigma}=A_{k}-l_{\sigma}$. In conclusion, we get $l_{\sigma} \leq \varepsilon$.

The case $k>i$ can be treated by duality, considering the group $A\left[\pi^{-}\right]$. We can also give a direct argument. Let us denote the group $L_{0} /\left(L_{0} \cap H_{A_{k}}\right)$ by $L_{k}^{\prime}$. Since $H_{A_{k}}$ and $L_{0}$ generate $A\left[\pi^{+}\right]$, the morphism $L_{k}^{\prime} \rightarrow A\left[\pi^{+}\right] / H_{A_{k}}$ is an isomorphism in generic fiber, so we get $\operatorname{deg} L_{k}^{\prime} \leq \operatorname{deg}\left(A\left[\pi^{+}\right] / H_{A_{k}}\right)$. But we have

$$
\operatorname{deg}\left(A\left[\pi^{+}\right] / H_{A_{k}}\right)=\operatorname{deg} A\left[\pi^{+}\right]-\operatorname{deg} H_{A_{k}} \leq \operatorname{deg} A\left[\pi^{+}\right]-d_{k}+\varepsilon .
$$

From Lemma 3.5, we get $\operatorname{deg}_{\sigma} L_{k}^{\prime} \leq \varepsilon$. Since $L_{0} \cap H_{A_{k}}$ is of height $f\left(A_{k}-A_{i}\right)$, we have

$$
l_{\sigma}=\operatorname{deg}_{\sigma} L_{0}-\left(A_{k}-A_{i}\right)=\operatorname{deg}_{\sigma} L_{k}^{\prime}+\operatorname{deg}_{\sigma}\left(L_{0} \cap H_{A_{k}}\right)-\left(A_{k}-A_{i}\right) \leq \varepsilon .
$$

Remark 3.7. Actually, we can have more control on the $l_{\sigma}$. Indeed, suppose that there exists $\sigma \in \Sigma_{k} \cap S_{1}$. If $k<i$, then we have $l_{\sigma} \leq \varepsilon$ for all $\sigma \in \Sigma_{j}$ with $j \leq k$. If $k>i$, then we have $l_{\sigma} \leq \varepsilon$ for all $\sigma \in \Sigma_{j}$ with $j \geq k$.

Putting together all the calculations made, we get the following result:

Proposition 3.8. We have

$$
\sum_{\sigma \in S_{2}} l_{\sigma} \geq 1-\alpha-f \varepsilon
$$

Proof. If $\sigma \in S_{1}$ we have $l_{\sigma} \leq \varepsilon$, and we also have $\sum_{\sigma \in \Sigma} l_{\sigma} \geq 1-\alpha$. Thus,

$$
\sum_{\sigma \in S_{2}} l_{\sigma} \geq 1-\alpha-\sum_{\sigma \in S_{1}} l_{\sigma} \geq 1-\alpha-f \varepsilon .
$$

We can now prove the bound for the norm of the operator $U_{i}^{\text {bad }}$.

Proposition 3.9. We have

$$
\left\|\alpha_{i}^{-1} U_{i}^{\mathrm{bad}}\right\| \leq p^{v\left(\alpha_{i}\right)+n_{A_{i}}-(1-\alpha-2 f \varepsilon) \inf _{\sigma \in S_{2}}\left(\kappa_{\sigma}+\lambda_{\sigma}\right)} .
$$

Proof. The term $p^{v\left(\alpha_{i}\right)}$ is the norm of the element $\alpha_{i}^{-1}$. Recall also the normalization factor for the Hecke operator,

$$
N_{A_{i}}=n_{A_{i}}+\sum_{\sigma \in \Sigma_{\pi}}\left(\max \left(a_{\sigma}-A_{i}, 0\right) \kappa_{\sigma}+\max \left(A_{i}-a_{\sigma}, 0\right) \lambda_{\sigma}\right) .
$$


The first term will come in the bound and the second term will be canceled out by the bounds for the partial degrees of $L_{0}$ and $L_{0}^{\perp}$.

Indeed, let us calculate the norm of the morphism $\omega_{A}^{\kappa} \rightarrow \omega_{A / L}^{\kappa}$. Let $\kappa_{1}$ be the weight defined by $\left(\left(\kappa_{\sigma}, \ldots, \kappa_{\sigma}\right),\left(\lambda_{\sigma}, \ldots, \lambda_{\sigma}\right)\right)_{\sigma \in \Sigma}$ and $\kappa_{2}=\kappa-\kappa_{1}$. Thus, $\omega^{\kappa}=\omega^{\kappa_{1}} \otimes \omega^{\kappa_{2}}$ and $\omega^{\kappa_{1}}$ is a line bundle. Since $\kappa_{2}$ has nonnegative coefficients, the morphism $\omega_{A}^{\kappa_{2}} \rightarrow \omega_{A / L}^{\kappa_{2}}$ has norm less than 1. It then suffices to study the morphism $\omega_{A}^{\kappa_{1}} \rightarrow \omega_{A / L}^{\kappa_{1}}$. But we have

$$
\omega_{A}^{\kappa_{1}}=\bigotimes_{\sigma \in \Sigma}\left(\operatorname{det} \omega_{A, \sigma}^{+}\right)^{\kappa_{\sigma}} \otimes\left(\operatorname{det} \omega_{A, \sigma}^{-}\right)^{\lambda_{\sigma}},
$$

where $\omega_{A, \sigma}^{+}$is the submodule of $\omega_{A}$ where $O_{F}$ acts on $\sigma^{+}$, and similarly for $\omega_{A, \sigma}^{-}$. We recall that $L=L_{0} \oplus L_{0}^{\perp}$. The norm of the map $\omega_{A}^{\kappa_{1}} \rightarrow \omega_{A / L}^{\kappa_{1}}$ is exactly $p^{A}$, with

$$
A=-\sum_{\sigma \in \Sigma}\left(\kappa_{\sigma} \operatorname{deg}_{\sigma} L_{0}+\lambda_{\sigma} \operatorname{deg}_{\sigma} L_{0}^{\perp}\right) .
$$

But recall that $\operatorname{deg}_{\sigma} L_{0}=\max \left(a_{\sigma}-A_{i}, 0\right)+l_{\sigma}$ and that

$$
\operatorname{deg}_{\sigma} L_{0}^{\perp}=A_{i}-a_{\sigma}+\operatorname{deg}_{\sigma} L_{0}=\max \left(A_{i}-a_{\sigma}, 0\right)+l_{\sigma} .
$$

Thus,

$$
\begin{aligned}
A & =-\sum_{\sigma \in \Sigma}\left(\max \left(a_{\sigma}-A_{i}, 0\right) \kappa_{\sigma}+\max \left(A_{i}-a_{\sigma}, 0\right) \lambda_{\sigma}+l_{\sigma}\left(\kappa_{\sigma}+\lambda_{\sigma}\right)\right) \\
& =-\left(N_{A_{i}}-n_{A_{i}}\right)+B
\end{aligned}
$$

with

$$
\begin{aligned}
B & =-\sum_{\sigma \in \Sigma}\left(l_{\sigma}\left(\kappa_{\sigma}+\lambda_{\sigma}\right)\right) \leq-\sum_{\sigma \in S_{2}}\left(l_{\sigma}\left(\kappa_{\sigma}+\lambda_{\sigma}\right)\right) \\
& \leq-\inf _{\sigma \in S_{2}}\left(\kappa_{\sigma}+\lambda_{\sigma}\right) \sum_{\sigma \in S_{2}} l_{\sigma} \leq-(1-\alpha-f \varepsilon) \inf _{\sigma \in S_{2}}\left(\kappa_{\sigma}+\lambda_{\sigma}\right) .
\end{aligned}
$$

Hence the bound for the operator $\alpha_{i}^{-1} U_{i}^{\text {bad }}$ follows, noting that $1-\alpha-f \varepsilon \geq$ $1-\alpha-2 f \varepsilon$.

Since we have made the assumption

$$
v\left(\alpha_{i}\right)+n_{A_{i}}<(1-2 f \varepsilon) \inf _{\sigma \in S_{2}}\left(\kappa_{\sigma}+\lambda_{\sigma}\right),
$$

we see that, if $\alpha$ is small enough, the norm of the operator $\alpha_{i}^{-1} U_{i}^{\text {bad }}$ is strictly less than 1 .

Remark 3.10. Actually, we only needed the weaker hypothesis

$$
v\left(\alpha_{i}\right)+n_{A_{i}}<(1-f \varepsilon) \inf _{\sigma \in S_{2}}\left(\kappa_{\sigma}+\lambda_{\sigma}\right),
$$

but we used ours to have the same condition in both the linear and unitary cases. 
3B2. Unitary case. We now turn to the case (U). We first assume that $i<\frac{1}{2}(a+b)$, so that the integer $t$ is equal to 1 . Let $M$ be a finite extension of $\mathbb{Q}_{p}$, let $x=$ $\left(A, \lambda, \iota, \eta, H_{\bullet}\right)$ be a point of $\mathcal{U}$ defined over $O_{M}$, and let $L$ be a totally isotropic $O_{F}$-stable subgroup of $A\left[\pi^{2}\right]$ such that $A[\pi]=H_{i} \oplus L[\pi]$. Let $A^{\prime}=A / L$, and let $H_{A_{i}}^{\prime}$ be the image of $H_{A_{i}}$ in $A / L$. Thus,

$$
H_{A_{i}}^{\prime}=(A[\pi]+L) / L .
$$

We suppose that the subgroup $L$ corresponds to a bad point, that is to say that $\operatorname{deg} H_{A_{i}}^{\prime} \leq d_{i}-1+\alpha$ for a certain rational $\alpha>0$. We write

$$
\operatorname{deg}_{\sigma_{1}} L=\max \left(a_{\sigma}-A_{i}, 0\right)+l_{\sigma}
$$

and

$$
\operatorname{deg}_{\sigma_{2}} L=b_{\sigma}-a_{\sigma}+\operatorname{deg}_{\sigma_{1}} L=\max \left(b_{\sigma}-A_{i}, b_{\sigma}-a_{\sigma}\right)+l_{\sigma}
$$

for all $\sigma \in \Sigma$. As it is shown by Proposition 2.10, $l_{\sigma}$ is a positive rational for all $\sigma$. We then have $\operatorname{deg} H_{A_{i}}^{\prime}=2 f A_{i}-\operatorname{deg}(L / L[\pi])$; therefore,

$$
\operatorname{deg}(L / L[\pi]) \geq 2 f A_{i}-d_{i}+1-\alpha .
$$

We also have, by Lemma 2.12, $\operatorname{deg}(L / L[\pi]) \leq 2 f A_{i}-\operatorname{deg} A[\pi]+\operatorname{deg} L[\pi]$. Thus,

$$
\operatorname{deg} L[\pi] \geq \operatorname{deg} A[\pi]-d_{i}+1-\alpha .
$$

We conclude that

$$
\operatorname{deg} L \geq 2 f A_{i}-2 d_{i}+\operatorname{deg} A[\pi]+2(1-\alpha) .
$$

By an explicit computation, one checks the equality

$$
2 f A_{i}-2 d_{i}+\operatorname{deg} A[\pi]=\sum_{\sigma \in \Sigma}\left(\max \left(a_{\sigma}-A_{i}, 0\right)+\max \left(b_{\sigma}-A_{i}, b_{\sigma}-a_{\sigma}\right)\right) .
$$

We conclude that the condition of being a bad point gives

$$
\sum_{\sigma \in \Sigma} l_{\sigma} \geq 1-\alpha
$$

Actually, we can control some of the $l_{\sigma}$. First, we prove the following technical lemma:

Lemma 3.11. Let $x=\left(A, \lambda, \iota, \eta, H_{\text {. }}\right)$ be a point as before, and let $H \subset A[\pi]$ be an $O_{F}$-stable subgroup. If $H$ is of height $2 f A_{k}$ and $\operatorname{deg} H \geq d_{k}-\varepsilon$, then $\operatorname{deg}_{\sigma_{1}} H \geq \min \left(a_{\sigma}, A_{k}\right)-\varepsilon$ for all $\sigma \in \Sigma$.

If $H$ is of height $2 f\left(a+b-A_{k}\right)$ and $\operatorname{deg} H \leq \operatorname{deg} A[\pi]-d_{k}+\varepsilon$, then $\operatorname{deg}_{\sigma_{1}} H \leq$ $\max \left(a_{\sigma}-A_{k}, 0\right)+\varepsilon$ for all $\sigma \in \Sigma$. 
Proof. Suppose that $H$ is of height $2 f A_{k}$ and $\operatorname{deg} H \geq d_{k}-\varepsilon$. If $\operatorname{deg}_{\sigma_{1}} H<$ $\min \left(a_{\sigma}, A_{k}\right)-\varepsilon$ for some $\sigma \in \Sigma$, then

$$
\begin{aligned}
\operatorname{deg} H & =\sum_{\sigma^{\prime} \in \Sigma}\left(\operatorname{deg}_{\sigma_{1}^{\prime}} H+\operatorname{deg}_{\sigma_{2}^{\prime}} H\right) \\
& <\min \left(a_{\sigma}, A_{k}\right)-\varepsilon+A_{k}+\sum_{\sigma^{\prime} \neq \sigma}\left(\min \left(a_{\sigma^{\prime}}, A_{k}\right)+A_{k}\right)=d_{k}-\varepsilon,
\end{aligned}
$$

and we get a contradiction.

If $H$ is of height $2 f\left(a+b-A_{k}\right)$ and $\operatorname{deg} H \leq \operatorname{deg} A[\pi]-d_{k}+\varepsilon$, then we can apply the previous argument to $A[\pi] / H$. We get $\operatorname{deg}_{\sigma_{1}}(A[\pi] / H) \geq \min \left(a_{\sigma}, A_{k}\right)-\varepsilon$ for all $\sigma \in \Sigma$, and therefore $\operatorname{deg}_{\sigma_{1}} H \leq \max \left(a_{\sigma}-A_{k}, 0\right)+\varepsilon$.

Now we prove a bound for some of the $l_{\sigma}$.

Lemma 3.12. If $\sigma \in S_{1}$, we have $l_{\sigma} \leq 2 \varepsilon$.

Proof. If $\sigma \in \Sigma_{0}$ then $l_{\sigma}=0$. If not, $\sigma \in \Sigma_{k}$ with $1 \leq k \leq s, k \neq i$, and $\varepsilon_{k}=\varepsilon$. Since $x$ is a point of $\mathcal{U}$, we have $\operatorname{deg} H_{A_{k}} \geq d_{k}-\varepsilon$. Suppose first that $k<i$. Since $H_{A_{k}}$ and $L[\pi]$ are disjoint, the morphism $H_{A_{k}} \rightarrow H_{A_{k}}^{\prime}:=\left(H_{A_{k}}+L[\pi]\right) / L[\pi]$ is an isomorphism in the generic fiber. Thus, by the properties of the degree function, $\operatorname{deg} H_{A_{k}}^{\prime} \geq \operatorname{deg} H_{A_{k}} \geq d_{k}-\varepsilon$. By Lemma 3.11, we have $\operatorname{deg}_{\sigma_{1}} H_{A_{k}}^{\prime} \geq$ $A_{k}-\varepsilon$. But we also have $\operatorname{deg}_{\sigma_{1}} H_{A_{k}}^{\prime} \leq \operatorname{deg}_{\sigma_{1}} A[\pi] / L[\pi]=a_{\sigma}-\operatorname{deg}_{\sigma_{1}} L[\pi]$, and therefore $\operatorname{deg}_{\sigma_{1}} L[\pi] \leq \varepsilon$. Next, we study $H_{A_{k}}^{\prime \prime}:=\left(H_{A_{k}}^{\prime}+L / L[\pi]\right) /(L / L[\pi])$. Using Lemma 3.11, we get

$$
A_{k}-\varepsilon \leq \operatorname{deg}_{\sigma_{1}} H_{A_{k}^{\prime \prime}} \leq A_{k}-\operatorname{deg}_{\sigma_{1}} L / L[\pi] .
$$

Therefore, $\operatorname{deg}_{\sigma_{1}}(L / L[\pi]) \leq \varepsilon$. In conclusion, we get $l_{\sigma} \leq 2 \varepsilon$.

The case $k>i$ cannot be treated by duality in this case. Let us denote the group $L[\pi] /\left(L[\pi] \cap H_{A_{k}}\right)$ by $L_{k}^{\prime}$. Since $H_{A_{k}}$ and $L[\pi]$ generate $A[\pi]$, the morphism $L_{k}^{\prime} \rightarrow A[\pi] / H_{A_{k}}$ is an isomorphism in generic fiber, so we get $\operatorname{deg} L_{k}^{\prime} \leq$ $\operatorname{deg}\left(A[\pi] / H_{A_{k}}\right)$. But we have

$$
\operatorname{deg}\left(A[\pi] / H_{A_{k}}\right)=\operatorname{deg} A[\pi]-\operatorname{deg} H_{A_{k}} \leq \operatorname{deg} A[\pi]-d_{k}+\varepsilon .
$$

Lemma 3.11 gives $\operatorname{deg}_{\sigma_{1}} L_{k}^{\prime} \leq \varepsilon$. Since $L[\pi] \cap H_{A_{k}}$ is of height $2 f\left(A_{k}-A_{i}\right)$, we have

$$
\operatorname{deg}_{\sigma_{1}} L[\pi]=\operatorname{deg}_{\sigma_{1}} L_{k}^{\prime}+\operatorname{deg}_{\sigma_{1}} L[\pi] \cap H_{A_{k}} \leq \varepsilon+A_{k}-A_{i} .
$$

Now we study $A^{\prime}:=A / L[\pi]$ and set $H_{A_{k}}^{\prime}:=\left(\pi^{-1}\left(H_{A_{k}} \cap L[\pi]\right)\right) / L[\pi]$. If $H_{A_{k}}^{\prime \prime}$ is the image of the morphism $H_{A_{k}}^{\prime} \rightarrow A^{\prime}[\pi] /(L / L[\pi])$, then we get deg $H_{A_{k}}^{\prime \prime} \geq d_{k}-\varepsilon$. Lemma 3.11 gives

$$
A_{k}-\varepsilon \leq \operatorname{deg}_{\sigma_{1}} H_{A_{k}}^{\prime \prime} \leq A_{k}-\operatorname{deg}_{\sigma_{1}}(L / L[\pi]) .
$$


We deduce that $\operatorname{deg}_{\sigma_{1}} L / L[\pi] \leq \varepsilon$. Finally, we have

$$
l_{\sigma}=\operatorname{deg}_{\sigma_{1}} L-\left(A_{k}-A_{i}\right) \leq 2 \varepsilon .
$$

Remark 3.13. Actually, we can have more control on the $l_{\sigma}$. Indeed, suppose that there exists $\sigma \in \Sigma_{k} \cap S_{1}$. If $k<i$, then we have $l_{\sigma} \leq \varepsilon$ for all $\sigma \in \Sigma_{j}$ with $j \leq k$. If $k>i$, then we have $l_{\sigma} \leq \varepsilon$ for all $\sigma \in \Sigma_{j}$ with $j \geq k$.

Putting together all the calculations made, we get the following result:

Proposition 3.14. We have

$$
\sum_{\sigma \in S_{2}} l_{\sigma} \geq 1-\alpha-2 f \varepsilon
$$

Proof. If $\sigma \in S_{1}$, we have $l_{\sigma} \leq 2 \varepsilon$, and we also have $\sum_{\sigma \in \Sigma} l_{\sigma} \geq 1-\alpha$. Thus,

$$
\sum_{\sigma \in S_{2}} l_{\sigma} \geq 1-\alpha-\sum_{\sigma \in S_{1}} l_{\sigma} \geq 1-\alpha-2 f \varepsilon .
$$

We can now prove the bound for the norm of the operator $U_{i}^{\text {bad }}$.

Proposition 3.15. We have

$$
\left\|\alpha_{i}^{-1} U_{i}^{\mathrm{bad}}\right\| \leq p^{v\left(\alpha_{i}\right)+n_{A_{i}}-(1-\alpha-2 f \varepsilon) \inf _{\sigma \in S_{2}}\left(\kappa_{\sigma}+\lambda_{\sigma}\right)} .
$$

Proof. The proof is exactly the same as in the linear case.

Since we have made the assumption

$$
v\left(\alpha_{i}\right)+n_{A_{i}}<(1-2 f \varepsilon) \inf _{\sigma \in S_{2}}\left(\kappa_{\sigma}+\lambda_{\sigma}\right),
$$

we see that, if $\alpha$ is small enough, the norm of the operator $\alpha_{i}^{-1} U_{i}^{\text {bad }}$ is strictly less than 1 .

We now deal with the case $i=\frac{1}{2}(a+b)$; the integer $t$ is now equal to 2 . The subgroup $L$ is now a maximal totally isotropic subgroup of $A[\pi]$. We write $\operatorname{deg}_{\sigma_{1}} L=l_{\sigma}$, and we have $\operatorname{deg}_{\sigma_{2}} L=\frac{1}{2}\left(b_{\sigma}-a_{\sigma}\right)+l_{\sigma}$ for all $\sigma \in \Sigma$. Recall that

$$
\operatorname{deg} H_{(a+b) / 2}^{\prime}=f(a+b)-\operatorname{deg} L=\sum_{\sigma \in \Sigma}\left(a_{\sigma}+\frac{1}{2}(a+b)-2 l_{\sigma}\right),
$$

so that

$$
d_{(a+b) / 2}-\operatorname{deg} H_{(a+b) / 2}^{\prime}=2 \sum_{\sigma \in \Sigma} l_{\sigma} .
$$

The condition of being a bad point gives

$$
\sum_{\sigma \in \Sigma} l_{\sigma} \geq 1-\alpha
$$

for a certain $\alpha>0$. The calculations are now exactly the same as previously, and the bound obtained in Proposition 3.15 is still valid in the case $i=\frac{1}{2}(a+b)$. 
3B3. Conclusion. We now recall the analytic continuation result we got, putting together all the results of the past sections.

Recall that we have defined subsets

$$
\begin{aligned}
\mathcal{U}_{0} & :=\operatorname{Deg}^{-1}\left(\left[d_{1}-\varepsilon_{1}, d_{1}\right] \times \cdots \times\left[d_{s}-\varepsilon_{s}, d_{s}\right]\right)=\bigcap_{k=1}^{s} \operatorname{Deg}_{k}^{-1}\left[d_{k}-\varepsilon_{k}, d_{k}\right], \\
\mathcal{U}_{1} & :=\bigcap_{k \neq i} \operatorname{Deg}_{k}^{-1}\left[d_{k}-\varepsilon_{k}, d_{k}\right] .
\end{aligned}
$$

We also have defined a partition $\Sigma=S_{1} \amalg S_{2}$. The result of this section is thus contained in the following theorem:

Theorem 3.16. Let $\kappa$ be a weight and $f$ a section of $\omega^{\kappa}$ on $\mathcal{U}_{0}$. Suppose that $f$ is an eigenform for the Hecke operators $U_{i}$, with eigenvalues $\alpha_{i}$, for all $1 \leq i \leq s$, and that we have the relations

$$
n_{A_{i}}+v\left(\alpha_{i}\right)<(1-2 f \varepsilon) \inf _{\sigma \in S_{2}}\left(\kappa_{\sigma}+\lambda_{\sigma}\right) .
$$

Then $f$ can be extended to a section of $\omega^{\kappa}$ on $\mathcal{U}_{1}$.

3C. The classicality theorem. We recall that we have assumed that $p$ is inert in $F_{0}$. We have defined integers $\left(A_{i}\right)_{1 \leq i \leq s}$, and to each of these integers corresponds a canonical subgroup and a relevant Hecke operator. We also have a partition

$$
\Sigma=\Sigma_{0} \amalg \coprod_{j=1}^{s} \Sigma_{j} \amalg \Sigma_{s+1} .
$$

We can now state the classicality result.

Theorem 3.17. Let $f$ be an overconvergent modular form of weight $\kappa$. Suppose that $f$ is an eigenform for the Hecke operators $U_{\pi, A_{i}}$, with eigenvalues $\alpha_{i}$, and that

$$
n_{A_{i}}+v\left(\alpha_{i}\right)<\inf _{\sigma \in \Sigma_{i}}\left(\kappa_{\sigma}+\lambda_{\sigma}\right)
$$

for $1 \leq i \leq s$. Then $f$ is classical.

Before giving the proof of the theorem, let us make the conditions explicit. In the case $(\mathrm{L})$, the conditions become

$$
v\left(\alpha_{i}\right)+\sum_{j=1}^{f} \min \left(a_{j}, A_{i}\right) \min \left(b_{j}, B_{i}\right)<\inf _{\sigma \in \Sigma_{i}}\left(\kappa_{\sigma}+\lambda_{\sigma}\right) .
$$

In the special case where all the $\left(a_{i}\right)$ are distinct and different from 0 and $a+b$, we have $s=d$ conditions, which can be written

$$
v\left(\alpha_{\sigma}\right)+\sum_{\sigma^{\prime} \in \Sigma} \min \left(a_{\sigma}, a_{\sigma^{\prime}}\right) \min \left(b_{\sigma}, b_{\sigma^{\prime}}\right)<\kappa_{\sigma}+\lambda_{\sigma},
$$

where $\alpha_{\sigma}$ is the eigenvalue of $U_{\pi, a_{\sigma}}$. 
In the case (U), they become

$$
v\left(\alpha_{i}\right)+\sum_{j=1}^{f}(a+b) \min \left(a_{j}, A_{i}\right)<\inf _{\sigma \in \Sigma_{i}}\left(\kappa_{\sigma}+\lambda_{\sigma}\right)
$$

if $A_{i}<\frac{1}{2}(a+b)$, and

$$
v\left(\alpha_{i}\right)+\sum_{j=1}^{f} \frac{1}{2}(a+b) a_{j}<\inf _{\sigma \in \Sigma_{i}}\left(\kappa_{\sigma}+\lambda_{\sigma}\right)
$$

if $A_{i}=\frac{1}{2}(a+b)$.

In the special case where all the $\left(a_{i}\right)$ are distinct and different from 0 and $\frac{1}{2}(a+b)$, we have $s=d$ conditions, which can be written

$$
v\left(\alpha_{\sigma}\right)+\sum_{\sigma^{\prime} \in \Sigma}(a+b) \min \left(a_{\sigma}, a_{\sigma^{\prime}}\right)<\kappa_{\sigma}+\lambda_{\sigma},
$$

where $\alpha_{\sigma}$ is the eigenvalue of $U_{\pi, a_{\sigma}}$.

Of course, in the case where the ordinary locus is nonempty, we find the same conditions as in [Bijakowski et al. 2016]. In the case (L), the condition of ordinariness is $a_{\sigma}=a, b_{\sigma}=b$ for some pair $(a, b)$ and for all $\sigma \in \Sigma$. There is one relevant Hecke operator, $U_{\pi, a}$, and the classicality condition is

$$
f a b+v(\alpha)<\inf _{\sigma \in \Sigma}\left(\kappa_{\sigma}+\lambda_{\sigma}\right),
$$

where $\alpha$ is the eigenvalue of $U_{\pi, a}$.

In the case (U), the condition of ordinariness is

$$
a_{\sigma}=b_{\sigma}=\frac{1}{2}(a+b)
$$

for all $\sigma \in \Sigma$. There is one relevant Hecke operator, $U_{\pi,(a+b) / 2}$, and the condition is

$$
\frac{1}{4} f(a+b)^{2}+v(\alpha)<\inf _{\sigma \in \Sigma}\left(\kappa_{\sigma}+\lambda_{\sigma}\right),
$$

where $\alpha$ is the eigenvalue of $U_{\pi,(a+b) / 2}$.

Remark 3.18. Since we need to use all the Hecke operators $U_{\pi, A_{i}}$ for the classicality result, maybe the relevant operator is a product of these ones. For example, in the linear case the operator $\prod_{\sigma} U_{\pi, a_{\sigma}}$ parametrizes complements of (a lifting of) the kernel of the $f$-th power of the Frobenius on the $\mu$-ordinary locus.

3C1. Proof of the theorem. The overconvergent form $f$ is a section of the sheaf $\omega^{\kappa}$ defined over

$$
\mathcal{V}_{0}:=\bigcap_{i=1}^{s} \operatorname{Deg}_{i}^{-1}\left(\left[d_{i}-\varepsilon, d_{i}\right]\right)
$$


for some $\varepsilon>0$. Of course, $\varepsilon$ can be taken as small as we want. Let us write $K_{i}=\inf _{\sigma \in \Sigma_{i}}\left(\kappa_{\sigma}+\lambda_{\sigma}\right)$ for all $1 \leq i \leq s$. We put the elements $\left(K_{i}\right)_{1 \leq i \leq s}$ in decreasing order:

$$
K_{i_{1}} \geq K_{i_{2}} \geq \cdots \geq K_{i_{s}}
$$

We will use the analytic continuation theorem successively for the operators $U_{i_{1}}, \ldots, U_{i_{s}}$, in that order.

We first consider the operator $U_{i_{1}}$ (we recall that we let $U_{i}:=U_{\pi, A_{i}}$ ). We take all the rationals $\varepsilon_{k}$ to be equal to $\varepsilon$. In that case, $S_{2}=\Sigma_{i_{1}}$. We can apply the analytic continuation theorem if the condition

$$
n_{A_{i_{1}}}+v\left(\alpha_{i_{1}}\right)<(1-2 f \varepsilon) \inf _{\sigma \in S_{2}}\left(\kappa_{\sigma}+\lambda_{\sigma}\right)
$$

is fulfilled. But $\inf _{\sigma \in S_{2}}\left(\kappa_{\sigma}+\lambda_{\sigma}\right)=K_{i_{1}}$ and we have, by hypothesis,

$$
n_{A_{i_{1}}}+v\left(\alpha_{i_{1}}\right)<K_{i_{1}} \text {. }
$$

If $\varepsilon$ is small enough, then we can apply the theorem. We can thus extend $f$ to

$$
\mathcal{V}_{1}:=\bigcap_{i \neq i_{1}} \operatorname{Deg}_{i}^{-1}\left(\left[d_{i}-\varepsilon, d_{i}\right]\right)
$$

We then use the operator $U_{i_{2}}$. In this case, we take all the rationals $\varepsilon_{k}$ to be equal to $\varepsilon$, except $\varepsilon_{i_{1}}=d_{i_{1}}$. In that case, $S_{2}=\Sigma_{i_{1}} \cup \Sigma_{i_{2}}$. We can apply the analytic continuation theorem if the condition

$$
n_{A_{i_{2}}}+v\left(\alpha_{i_{2}}\right)<(1-2 f \varepsilon) \inf _{\sigma \in S_{2}}\left(\kappa_{\sigma}+\lambda_{\sigma}\right)
$$

is fulfilled. But $\inf _{\sigma \in S_{2}}\left(\kappa_{\sigma}+\lambda_{\sigma}\right)=\inf \left(K_{i_{1}}, K_{i_{2}}\right)=K_{i_{2}}$ and we have, by hypothesis,

$$
n_{A_{i_{2}}}+v\left(\alpha_{i_{2}}\right)<K_{i_{2}} \text {. }
$$

If $\varepsilon$ is small enough, then we can apply the theorem. We extend $f$ to

$$
\mathcal{V}_{2}:=\bigcap_{i \notin\left\{i_{1}, i_{2}\right\}} \operatorname{Deg}_{i}^{-1}\left(\left[d_{i}-\varepsilon, d_{i}\right]\right) .
$$

Repeating this argument, we can extend the overconvergent form $f$ to the whole

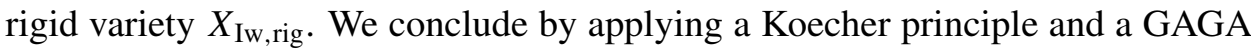
theorem, which proves that the space

$$
H^{0}\left(X_{\mathrm{Iw}, \mathrm{rig}}, \omega^{\kappa}\right)
$$

consists of classical modular forms. This will be done in the next section. 
3C2. Compactifications and Koecher's principle. To complete the proof of the theorem, we need to prove a Koecher principle and to introduce compactifications of the Shimura variety.

Proposition 3.19. There exists a toroidal compactification $\bar{X}_{\mathrm{Iw}}$ of $X_{\mathrm{Iw}}$ defined over $O_{K}$. It is a proper scheme, and the sheaf $\omega^{\kappa}$ extends to $\bar{X}_{\mathrm{Iw}}$.

The construction of the compactification of $X$ has been done in [Lan 2008], and the one for $X_{\text {Iw }}$ follows from [Lan 2015, Section 3] (see also [Bijakowski et al. 2016, Section 5.1]). One can also construct the minimal compactification $X_{\mathrm{Iw}}^{*}$ of $X_{\mathrm{Iw}}$. The Koecher principle states that, under a certain condition, the sections on $X_{\text {Iw }}$ automatically extend to the toroidal compactification. The next proposition follows from [Lan 2015, Theorem 8.7] (see also [Bijakowski et al. 2016, Section 5.2]).

Proposition 3.20. Suppose that the codimension of the boundary of $X_{\mathrm{I}}^{*}$ is greater than 2. Then for any $O_{K}$-algebra $R$ the restriction map

$$
H^{0}\left(\bar{X}_{\text {Iw }} \times R, \omega^{\kappa}\right) \rightarrow H^{0}\left(X_{\text {Iw }} \times R, \omega^{\kappa}\right)
$$

is an isomorphism.

From this, we deduce a rigid Koecher principle (under the same dimension assumption):

$$
H^{0}\left(\bar{X}_{\text {Iw }, \text { rig }}, \omega^{\kappa}\right) \simeq H^{0}\left(X_{\text {Iw,rig }}, \omega^{\kappa}\right),
$$

where $\bar{X}_{\text {Iw,rig }}$ is the rigid space associated to $\bar{X}_{\text {Iw }}$. Finally, since $\bar{X}_{\text {Iw }}$ is proper, we have a GAGA theorem.

Proposition 3.21. The analytification morphism

$$
H^{0}\left(\bar{X}_{\text {Iw }} \times K, \omega^{\kappa}\right) \rightarrow H^{0}\left(\bar{X}_{\text {Iw,rig }}, \omega^{\kappa}\right)
$$

is an isomorphism.

To conclude, we need to make explicit the dimension condition. We have the following cases:

- If there exists $\sigma$ such that $a_{\sigma} b_{\sigma}=0$, then the varieties $X$ and $X_{\text {Iw }}$ are compact [Lan 2008, Remark 5.3.3.2].

- If this is not the case, the codimension of the boundary of $X_{\mathrm{Iw}}^{*}$ is equal to $d(a+b-1)$. Indeed, the dimension of the variety is equal to $\sum_{\sigma} a_{\sigma} b_{\sigma}$. From [Lan 2008, Theorem 7.2.4.1], there is a stratification of $X_{\mathrm{Iw}}^{*}$, and any top-dimensional strata of the boundary is isomorphic to a Shimura variety with signatures $\left(a_{\sigma}-1, b_{\sigma}-1\right)$, hence has dimension $\sum_{\sigma}\left(a_{\sigma}-1\right)\left(b_{\sigma}-1\right)$.

If $d>1$ or $a+b \geq 3$, then the condition for the Koecher principle is fulfilled.

- If $d=a=b=1$, we cannot apply the Koecher principle. 
Since we have excluded the third case by Hypothesis 1.3, it follows from what has been said before that we have an isomorphism

$$
H^{0}\left(\bar{X}_{\mathrm{Iw}} \times K, \omega^{\kappa}\right) \simeq H^{0}\left(X_{\mathrm{Iw}, \text { rig }}, \omega^{\kappa}\right),
$$

that is to say that the space $H^{0}\left(X_{\mathrm{Iw}, \text { rig }}, \omega^{\kappa}\right)$ consists of classical modular forms. If the variety is compact, this is only the GAGA theorem, and in the noncompact case it is a combination of the rigid Koecher principle and the GAGA theorem. This concludes the proof of the theorem.

In the exceptional remaining case, the variety is essentially a modular curve. To prove the classicality theorem, one has to take the cusps into account in the series constructed. We refer to [Kassaei 2006] for more details.

\section{The case with several primes above $p$}

In this section, we no longer assume that there is only one prime of $F_{0}$ above $p$. We will define the degree functions and the overconvergent modular forms, and prove the classicality result.

Let $\mathcal{P}$ be the set of primes of $F_{0}$ above $p$. We write $\mathcal{P}=\left\{\pi_{1}, \ldots, \pi_{g}\right\}$. Recall that we have defined the Shimura variety of Iwahori level at $p$

$$
X_{\mathrm{Iw}}=X_{\mathrm{Iw}, \pi_{1}} \times_{X} X_{\mathrm{Iw}, \pi_{2}} \times_{X} \cdots \times_{X} X_{\mathrm{Iw}, \pi_{g}} .
$$

We will denote by $X_{\text {Iw,rig }}$ the rigid space associated to the scheme $X_{\text {Iw }}$. Let $\pi$ be an element of $\mathcal{P}$. In the previous sections, we have defined an integer $s_{\pi}$, integers $A_{\pi, 1}<\cdots<A_{\pi, s_{\pi}}$, other integers $d_{\pi, 1}, \ldots, d_{\pi, s_{\pi}}$, together with a degree function

$$
\operatorname{Deg}_{\pi}: X_{\mathrm{Iw}, \pi, \mathrm{rig}} \rightarrow \prod_{i=1}^{s_{\pi}}\left[0, d_{\pi, i}\right] .
$$

We will define the degree function on $X_{\text {Iw,rig }}$ by

$$
\begin{aligned}
\operatorname{Deg}: X_{\mathrm{Iw}, \mathrm{rig}} & \rightarrow \prod_{\pi \in \mathcal{P}} \prod_{i=1}^{s_{\pi}}\left[0, d_{\pi, i}\right], \\
x & \mapsto\left(\operatorname{Deg}_{\pi}\left(p_{\pi}(x)\right)\right)_{\pi \in \mathcal{P}} .
\end{aligned}
$$

Here $p_{\pi}$ is the projection $X_{\mathrm{Iw}, \text { rig }} \rightarrow X_{\mathrm{Iw}, \pi, \text { rig }}$.

Let $X_{\mathrm{Iw}}^{\mu \text {-ord-mult }}$ be the space $\operatorname{Deg}^{-1}\left(\left\{d_{\pi, i}\right\}\right)$. Let us fix a weight $\kappa$.

Definition 4.1. The space of overconvergent modular forms of weight $\kappa$ is defined as

$$
M^{\dagger}:=\operatorname{colim}_{\mathcal{V}} H^{0}\left(\mathcal{V}, \omega^{\kappa}\right),
$$

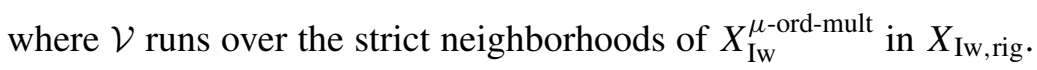


We have defined Hecke operators $U_{\pi, A_{\pi, 1}}, \ldots, U_{\pi, A_{\pi, s_{\pi}}}$. There is a slight ambiguity for the polarization $\lambda^{\prime}$. If $\pi$ is the only place above $p$, one can take $p \cdot \lambda$, which is a prime-to- $p$ polarization. In general, $\lambda^{\prime}$ is not well defined. Let $x$ be a totally positive element of $O_{F}$ such that $v_{\pi^{\prime}}(x)$ equals 1 if $\pi^{\prime}=\pi$, and 0 if $\pi^{\prime}$ is a place of $F_{0}$ above $p$ different from $\pi$. Then one takes for $\lambda^{\prime}$ the polarization $x \cdot \lambda$.

These operators act both on classical and overconvergent modular forms. We can finally state and prove the classicality theorem.

Theorem 4.2. Let $f$ be an overconvergent modular form. Suppose that $f$ is an eigenform for the Hecke operators $U_{\pi, A_{\pi, i}}$, with eigenvalues $\alpha_{\pi, i}$. If we have the relations

$$
n_{\pi, A_{\pi, i}}+v\left(\alpha_{\pi, i}\right)<\inf _{\sigma \in \Sigma_{\pi, i}}\left(\kappa_{\sigma}+\lambda_{\sigma}\right)
$$

for all $\pi \in \mathcal{P}, 1 \leq i \leq s_{\pi}$, then $f$ is classical.

We recall that $n_{\pi, i}$ is the constant term of the normalization factor for the Hecke operator $U_{\pi, i}$ and that $\Sigma_{\pi, i}=\left\{\sigma \in \Sigma_{\pi}: a_{\sigma}=A_{\pi, i}\right\}$.

Proof. We will use for each place $\pi$ the analytic continuation theorem we got in the previous section to extend the modular form to the whole rigid variety, and deduce its classicality. Luckily, the order of the places $\pi$ is not important here.

We start with an overconvergent form $f$. It is a section of $\omega^{\kappa}$ defined on a set of the form

$$
\operatorname{Deg}^{-1}\left(\prod_{\pi \in \mathcal{P}} \prod_{i=1}^{s_{\pi}}\left[d_{\pi, i}-\varepsilon, d_{\pi, i}\right]\right) .
$$

First, we consider the place $\pi_{1}$. Using the Hecke operators and the relations satisfied by the eigenvalues, by the previous section one can extend $f$ to

$$
\operatorname{Deg}^{-1}\left(\prod_{i=1}^{s_{\pi_{1}}}\left[0, d_{\pi_{1}, i}\right] \times \prod_{\pi \neq \pi_{1}} \prod_{i=1}^{s_{\pi}}\left[d_{\pi, i}-\varepsilon, d_{\pi, i}\right]\right) .
$$

Using this argument successively with the different places, one can extend $f$ to the whole rigid variety $X_{\text {Iw,rig. Using a Koecher principle (if the variety is not compact) }}$ and a GAGA theorem as in Section 3C2, one can prove that $f$ is a classical modular form.

\section{Acknowledgement}

The author would like to thank Benoît Stroh, Valentin Hernandez and Vincent Pilloni for helpful discussions. 


\section{References}

[Berthelot 1996] P. Berthelot, "Cohomologie rigide et cohomologie à supports propres, première partie", preprint IRMAR 96-03, 1996, available at https://perso.univ-rennes1.fr/pierre.berthelot/ publis/Cohomologie_Rigide_I.pdf.

[Bijakowski 2014] S. Bijakowski, Classicité de formes modulaires surconvergentes sur une variété de Shimura, Ph.D. thesis, Université Paris-Nord, 2014, available at https://tel.archives-ouvertes.fr/ tel-01275872.

[Bijakowski et al. 2016] S. Bijakowski, V. Pilloni, and B. Stroh, "Classicité de formes modulaires surconvergentes", Ann. Math. 183:3 (2016), 975-1014.

[Buzzard 2003] K. Buzzard, "Analytic continuation of overconvergent eigenforms", J. Amer. Math. Soc. 16:1 (2003), 29-55. MR Zbl

[Coleman 1996] R. F. Coleman, "Classical and overconvergent modular forms", Invent. Math. 124:1-3 (1996), 215-241. MR Zbl

[Fargues 2010] L. Fargues, "La filtration de Harder-Narasimhan des schémas en groupes finis et plats", J. Reine Angew. Math. 645 (2010), 1-39. MR Zbl

[Fargues 2011] L. Fargues, "La filtration canonique des points de torsion des groupes $p$-divisibles", Ann. Sci. École Norm. Sup. (4) 44:6 (2011), 905-961. MR Zbl

[Johansson 2013] C. Johansson, "Classicality for small slope overconvergent automorphic forms on some compact PEL Shimura varieties of type C", Math. Ann. 357:1 (2013), 51-88. MR Zbl

[Kassaei 2006] P. L. Kassaei, "A gluing lemma and overconvergent modular forms", Duke Math. J. 132:3 (2006), 509-529. MR Zbl

[Kottwitz 1992] R. E. Kottwitz, "Points on some Shimura varieties over finite fields", J. Amer. Math. Soc. 5:2 (1992), 373-444. MR Zbl

[Lan 2008] K.-W. Lan, Arithmetic compactifications of PEL-type Shimura varieties, thesis, Harvard University, 2008, available at http://search.proquest.com/docview/304599957. MR Zbl

[Lan 2015] K.-W. Lan, "Integral models of toroidal compactifications with projective cone decompositions", preprint, 2015, available at http://www.math.umn.edu/ kwlan/articles/cpt-ram-nbl.pdf.

[Moonen 2004] B. Moonen, "Serre-Tate theory for moduli spaces of PEL type", Ann. Sci. École Norm. Sup. (4) 37:2 (2004), 223-269. MR Zbl

[Pilloni 2011] V. Pilloni, "Prolongement analytique sur les variétés de Siegel", Duke Math. J. 157:1 (2011), 167-222. MR Zbl

[Pilloni and Stroh 2011] V. Pilloni and B. Stroh, "Surconvergence et classicité: le cas Hilbert", preprint, 2011, available at http://perso.ens-lyon.fr/vincent.pilloni/surconv_hilbert.pdf.

[Sasaki 2010] S. Sasaki, "Analytic continuation of overconvergent Hilbert eigenforms in the totally split case”, Compos. Math. 146:3 (2010), 541-560. MR Zbl

[Tian and Xiao 2013] Y. Tian and L. Xiao, " $p$-adic cohomology and classicality of overconvergent Hilbert modular forms", preprint, 2013. To appear in Arithmétique p-adique des formes de Hilbert, Astérisque. arXiv

[Wedhorn 1999] T. Wedhorn, "Ordinariness in good reductions of Shimura varieties of PEL-type", Ann. Sci. École Norm. Sup. (4) 32:5 (1999), 575-618. MR Zbl

Communicated by Brian Conrad

Received 2015-05-29 Revised 2016-04-11 Accepted 2016-05-12

s.bijakowski@imperial.ac.uk Department of Mathematics, Imperial College, 180 Queen's Gate, London, SW7 2AZ, United Kingdom 


\section{Algebra \& Number Theory}

msp.org/ant

\section{EDITORS}

MANAGING EDITOR

Bjorn Poonen

Massachusetts Institute of Technology

Cambridge, USA

\author{
EDITORIAL BOARD CHAIR \\ David Eisenbud \\ University of California \\ Berkeley, USA
}

BOARD OF EDITORS

Georgia Benkart

Dave Benson

Richard E. Borcherds

John H. Coates

J-L. Colliot-Thélène

Brian D. Conrad

Hélène Esnault

Hubert Flenner

Sergey Fomin

Edward Frenkel

Andrew Granville

Joseph Gubeladze

Roger Heath-Brown

Craig Huneke

Kiran S. Kedlaya

János Kollár

Yuri Manin

Philippe Michel
University of Wisconsin, Madison, USA

University of Aberdeen, Scotland

University of California, Berkeley, USA

University of Cambridge, UK

CNRS, Université Paris-Sud, France

Stanford University, USA

Freie Universität Berlin, Germany

Ruhr-Universität, Germany

University of Michigan, USA

University of California, Berkeley, USA

Université de Montréal, Canada

San Francisco State University, USA

Oxford University, UK

University of Virginia, USA

Univ. of California, San Diego, USA

Princeton University, USA

Northwestern University, USA

École Polytechnique Fédérale de Lausanne
Susan Montgomery

Shigefumi Mori

Raman Parimala

Jonathan Pila

Anand Pillay

Victor Reiner

Peter Sarnak

Joseph H. Silverman

Michael Singer

Vasudevan Srinivas

J. Toby Stafford

Ravi Vakil

Michel van den Bergh

Marie-France Vignéras

Kei-Ichi Watanabe

Efim Zelmanov

Shou-Wu Zhang
University of Southern California, USA

RIMS, Kyoto University, Japan

Emory University, USA

University of Oxford, UK

University of Notre Dame, USA

University of Minnesota, USA

Princeton University, USA

Brown University, USA

North Carolina State University, USA

Tata Inst. of Fund. Research, India

University of Michigan, USA

Stanford University, USA

Hasselt University, Belgium

Université Paris VII, France

Nihon University, Japan

University of California, San Diego, USA

Princeton University, USA

\section{PRODUCTION}

production@msp.org

Silvio Levy, Scientific Editor

See inside back cover or msp.org/ant for submission instructions.

The subscription price for 2016 is US $\$ 290 /$ year for the electronic version, and $\$ 485 /$ year ( $+\$ 55$, if shipping outside the US) for print and electronic. Subscriptions, requests for back issues and changes of subscribers address should be sent to MSP.

Algebra \& Number Theory (ISSN 1944-7833 electronic, 1937-0652 printed) at Mathematical Sciences Publishers, 798 Evans Hall \#3840, c/o University of California, Berkeley, CA 94720-3840 is published continuously online. Periodical rate postage paid at Berkeley, CA 94704, and additional mailing offices.

ANT peer review and production are managed by EditFLOW ${ }^{\circledR}$ from MSP.

\section{PUBLISHED BY}

- mathematical sciences publishers

nonprofit scientific publishing

http://msp.org/

(C) 2016 Mathematical Sciences Publishers 


\section{Algebra \& Number Theory}

Volume $10 \quad$ No. $4 \quad 2016$

Moduli of morphisms of logarithmic schemes

JONATHAN WISE

Residual intersections and the annihilator of Koszul homologies

SEYED HAMID HaSSANZADEH and Jose NAÉLITON

The Prym map of degree-7 cyclic coverings

HERBERT LANGE and ANGELA ORTEGA

SIMON MARSHALL

Hasse principle for Kummer varieties

Yonatan HaRPaZ and AlEXEI N. SkoRobogatov

Analytic continuation on Shimura varieties with $\mu$-ordinary locus

STÉPHANE BIJAKOWSKI

A note on secondary $K$-theory

GONÇALO TABUADA

Nef cones of Hilbert schemes of points on surfaces

Barbara Bolognese, Jack Huizenga, Yinbang Lin, Eric Riedl,

BENJAMIN SCHMidT, MATTHEW WoOlF and XiaOlei ZHAO

Interpolation for restricted tangent bundles of general curves 